\title{
Time-Inhomogeneous Feller-type Diffusion Process with Absorbing Boundary Condition
}

\author{
Virginia Giorno ${ }^{1}$ (D) Amelia G. Nobile ${ }^{1}$ (D)
}

Received: 22 December 2020 / Accepted: 12 May 2021 / Published online: 4 June 2021

(c) The Author(s) 2021

\begin{abstract}
A time-inhomogeneous Feller-type diffusion process with linear infinitesimal drift $\alpha(t) x+$ $\beta(t)$ and linear infinitesimal variance $2 r(t) x$ is considered. For this process, the transition density in the presence of an absorbing boundary in the zero-state and the first-passage time density through the zero-state are obtained. Special attention is dedicated to the proportional case, in which the immigration intensity function $\beta(t)$ and the noise intensity function $r(t)$ are connected via the relation $\beta(t)=\xi r(t)$, with $0 \leq \xi<1$. Various numerical computations are performed to illustrate the effect of the parameters on the first-passage time density, by assuming that $\alpha(t), \beta(t)$ or both of these functions exhibit some kind of periodicity.
\end{abstract}

Keywords Transient distributions · First-passage time densities $\cdot$ Periodic intensity functions

Mathematics Subject Classification $60 \mathrm{~J} 60 \cdot 60 \mathrm{~J} 70 \cdot 82 \mathrm{C} 31$

\section{Introduction and Background}

One-dimensional time-inhomogeneous diffusion processes play a relevant role in different application fields, including physics, biology, neuroscience, finance and others (cf., for instance, Giorno and Nobile [1,2], Albano and Giorno [3], Ghost and Prajneshu [4], Buonocore et al [5], Gutiérrez et al [6], Di Crescenzo et al [7], Román-Román et al. [8], Molini et al. [9], Gan and Waxman [10], Abundo [11]). In this paper, we consider a timeinhomogeneous Feller-type diffusion process, characterized by linear infinitesimal drift and linear infinitesimal variance vanishing in the zero-state (lower boundary of the process). We assume that the zero-state represents an absorbing boundary for the process.

Communicated by Christian Maes.

$凶$ Amelia G. Nobile

nobile@unisa.it

Virginia Giorno

giorno@unisa.it

1 Dipartimento di Informatica, Università degli Studi di Salerno, Via Giovanni Paolo II n. 132, 84084

Fisciano, SA, Italy 
Let $\left\{X(t), t \geq t_{0}\right\}, t_{0} \geq 0$, be a time-inhomogeneous Feller-type diffusion process with the following infinitesimal drift and infinitesimal variance

$$
A_{1}(x, t)=\alpha(t) x+\beta(t), \quad A_{2}(x, t)=2 r(t) x,
$$

defined in the state-space $[0,+\infty)$, with $\alpha(t) \in \mathbb{R}, \beta(t) \in \mathbb{R}, r(t)>0$ continuous functions for all $t \geq t_{0}$.

The time-homogeneous Feller diffusion process, in which $\alpha(t)=\alpha, \beta(t)=\beta$ and $r(t)=r$ for all $t \geq 0$, is taken in account in Feller [12], where is shown that boundary $x=0$ changes its character depending on whether $\beta \leq 0$ (exit), $0<\beta<r$ (regular), $\beta \geq r$ (entrance). Furthermore, as proved in Feller [13], if one knows the nature of the end points of the state-space one can decide what kind of boundary condition has to be associated with the Fokker-Planck and Kolmogorov diffusion equations to determine the transition pdf of the process. A review showing the relevance of the Feller's work on boundary classification of one-dimensional diffusion processes is provided in Peskir [14]. By following this approach, for the time-homogeneous Feller diffusion process, the transition pdf in the presence of an absorption condition or a zero-flux condition in the zero-state is explicitly obtained in Karlin and Taylor [15] and in Giorno et al. [16]. Furthermore, a class of Kolmogorov diffusion equations that can be transformed into a Kolmogorov equation for a time-homogeneous Feller process is considered in Capocelli and Ricciardi [17].

Feller diffusion process is widely used in mathematical biology to model the growth of a population (cf., Lavigne and Roques [18], Masoliver [19], Ricciardi et al. [20]), in queueing systems to describe the number of customers in a queue (cf., Di Crescenzo and Nobile [21]), in neurobiology to analyze the input-output behavior of single neurons (see, for instance, Ditlevsen and Lánský [22], Lánský et al. [23], Nobile and Pirozzi [24], Giorno et al. [25,26], Buonocore et al. [27]), in mathematical finance to model asset prices, market indices, interest rates and stochastic volatility (see, Tian and Zhang [28], Cox et al. [29], Linetsky [30], GöingJaeshke and Yor [31]).

Sometimes, the Feller-type diffusion process $X(t)$ is obtained as a continuous approximation of a time-inhomogeneous discrete Markov processes (see, for instance, Di Crescenzo and Nobile [21], Giorno et al. [25]). Indeed, in population dynamics the Feller-type diffusion process arises as a continuous approximation of a birth-death process with immigration (cf. Giorno and Nobile [32] and references therein). In these cases $\alpha(t)$, related to the growth intensity function, is positive (negative) when the birth intensity function is greater (less) than the death intensity function, whereas $\alpha(t)=0$ if the birth intensity function is equal to the death intensity function. Since $\alpha(t)$ is a time dependent function, it can be positive, negative or zero at different time instants. Instead, $\beta(t)$ is related to the immigration intensity function. In particular, $\beta(t)>0$ indicates the presence of immigrations and a zero-flux condition or an absorbing condition can be imposed in the zero-state of the diffusion process.

For a full characterization of the time-inhomogeneous Feller-type diffusion process $X(t)$, the behavior at the boundary 0 must be specified. In this paper, we assume that the zero-state is an absorbing boundary, so that the process $X(t)$ terminates when the boundary is reached. We suppose that $\alpha(t) \in \mathbb{R}, \beta(t) \in \mathbb{R}, r(t)>0, \beta(t) \leq \xi r(t)$, with $0 \leq \xi<1$, for all $t \geq t_{0}$.

We denote by

$$
f_{a}\left(x, t \mid x_{0}, t_{0}\right)=\frac{\partial}{\partial x} P\left\{X(t) \leq x ; X(\vartheta)>0, \forall \vartheta<t \mid X\left(t_{0}\right)=x_{0}\right\}, \quad x>0, y>0
$$

the transition probability density function (pdf) of $X(t)$ in the presence of an absorbing boundary in the zero-state. As shown in Kolmogorov [33] and Dynkin [34], the pdf $f_{a}\left(x, t \mid x_{0}, t_{0}\right)$ 
satisfies the Kolmogorov equation

$$
\frac{\partial f_{a}\left(x, t \mid x_{0}, t_{0}\right)}{\partial t_{0}}+A_{1}\left(x_{0}, t_{0}\right) \frac{\partial f_{a}\left(x, t \mid x_{0}, t_{0}\right)}{\partial x_{0}}+\frac{1}{2} A_{2}\left(x_{0}, t_{0}\right) \frac{\partial^{2} f_{a}\left(x, t \mid x_{0}, t_{0}\right)}{\partial x_{0}^{2}}=0,
$$

with $A_{1}\left(x_{0}, t_{0}\right)$ and $A_{2}\left(x_{0}, t_{0}\right)$ given in (1), to solve imposing the initial delta condition

$$
\lim _{t_{0} \uparrow t} f_{a}\left(x, t \mid x_{0}, t_{0}\right)=\delta\left(x-x_{0}\right)
$$

and the absorbing boundary condition in the zero-state:

$$
\lim _{x_{0} \downarrow 0} f_{a}\left(x, t \mid x_{0}, t_{0}\right)=0 .
$$

Furthermore, let

$$
T\left(x_{0}, t_{0}\right)=\inf _{t \geq t_{0}}\{t: X(t)=0\}, \quad X\left(t_{0}\right)=x_{0}>0
$$

be the random variable describing the first-passage time (FPT) through the zero-state starting from $X\left(t_{0}\right)=x_{0}>0$; we denote by

$$
g\left(0, t \mid x_{0}, t_{0}\right)=\frac{d}{d t} P\left\{T\left(x_{0}, t_{0}\right) \leq t\right\} .
$$

We note that the FPT density $g\left(0, t \mid x_{0}, t_{0}\right)$ is not affected by the boundary condition on the zero-state, provided that it is attainable.

The problem of determining FPT densities for the Feller-type diffusion process arises in a variety of fields, including neurobiology, population dynamics, queueing systems and mathematical finance (cf., for instance, Linetsky [30], Masoliver and Perelló [35], Buonocore et al. [36], D’Onofrio et al. [37], Giorno et al. [38,39], Albano e Giorno [40], Di Nardo and D'Onofrio [41]). For instance, in population dynamics $g\left(0, t \mid x_{0}, t_{0}\right)$ describes the extinction density, whereas in queueing systems represents the busy period density. Lavigne and Roques in [18] focus on the distribution of the extinction times of a population whose size is described by a time-inhomogeneous Feller-type diffusion process with infinitesimal drift $A_{1}(x, t)=$ $\alpha(t) x$ and infinitesimal variance $A_{2}(x, t)=\sigma^{2} x$, where $\alpha(t)$ is a continuous function and $\sigma^{2}$ is a positive constant.

The functions (2) and (7) are intimately related; indeed, one has:

$$
\int_{0}^{+\infty} f_{a}\left(x, t \mid x_{0}, t_{0}\right) d x+\int_{t_{0}}^{t} g\left(0, \tau \mid x_{0}, t_{0}\right) d \tau=1 .
$$

Relation (8) shows that the determination of $g\left(0, t \mid x_{0}, t_{0}\right)$ requires the explicit evaluation of the transition pdf $f_{a}\left(x, t \mid x_{0}, t_{0}\right)$ in the presence of an absorbing boundary at the zero-state.

\section{Plain of the Paper}

The paper is organized in five sections and seven appendices in which the proofs of the main results are reported. In Sect. 2, for the time-inhomogeneous Feller-type diffusion process $X(t)$, with infinitesimal moments (1), we give some preliminary results concerning the Laplace transform (according to $\left.x_{0}\right)$ of the transition pdf $f_{a}\left(x, t \mid x_{0}, t_{0}\right)$ in the presence of an absorbing boundary in the zero-state. The proportional case, in which the immigration intensity function $\beta(t)$ and the noise intensity function $r(t)$ are related as $\beta(t)=\xi r(t)$, with $0 \leq \xi<1$, is also analyzed. In Sect. 3, the transition pdf $f_{a}\left(x, t \mid x_{0}, t_{0}\right)$ is obtained for the process (1) in the general case, by distinguishing the case $x=0$ (Sect. 3.1) and $x>0$ 
(Sect. 3.2). In Sect. 4, we focus on the FPT of $X(t)$ through the zero-state for the general case and we determine the expression of the FPT pdf $g\left(0, t \mid x_{0}, t_{0}\right)$. In Sects. 3 and 4 , we also show as the results of the proportional case can be derived from the general case. In Sect. 5, various numerical computations are performed making use of MATHEMATICA to illustrate the effect of periodic intensity functions on the FPT pdf $g\left(0, t \mid x_{0}, t_{0}\right)$. Specifically, we assume that the growth intensity function $\alpha(t)$, the immigration intensity function $\beta(t)$ or both these functions exhibit some kind of periodicity. The FPT mean $t_{1}\left(0, t \mid x_{0}, t_{0}\right)$ and the coefficient of variation $\operatorname{CV}\left(0 \mid x_{0}, t_{0}\right)=\sqrt{\operatorname{Var}\left(0 \mid x_{0}, t_{0}\right)} / t_{1}\left(0 \mid x_{0}, t_{0}\right)$ are also analyzed.

\section{Preliminary Results}

In this section, we determine the Laplace transform (according to $x_{0}$ ) of the transition pdf $f_{a}\left(x, t \mid x_{0}, t_{0}\right)$ in the general case. Furthermore, the explicit expressions of the transition pdf and of the FPT density through the zero-state are obtained in the proportional case.

\subsection{Laplace Transform}

For $t \geq t_{0}$ and $x \geq 0$, we consider the Laplace transform:

$$
Z_{a}\left(x, t \mid s, t_{0}\right)=\int_{0}^{+\infty} e^{-s x_{0}} f_{a}\left(x, t \mid x_{0}, t_{0}\right) d x_{0}, \quad \operatorname{Re} s>0 .
$$

We determine $Z_{a}\left(x, t \mid s, t_{0}\right)$ so that, by taking its inverse Laplace transform, we obtain $f_{a}\left(x, t \mid x_{0}, t_{0}\right)$. Multiplying both sides of (3) by $e^{-s x_{0}}$, integrating with respect to $x_{0}$ over the interval $[0,+\infty)$ and making use of the boundary condition (5), we have the following partial differential equation

$$
\begin{aligned}
& \frac{\partial Z_{a}\left(x, t \mid s, t_{0}\right)}{\partial t_{0}}-s\left[\alpha\left(t_{0}\right)+s r\left(t_{0}\right)\right] \frac{\partial Z_{a}\left(x, t \mid s, t_{0}\right)}{\partial s} \\
& \quad+\left[s \beta\left(t_{0}\right)-\alpha\left(t_{0}\right)-2 s r\left(t_{0}\right)\right] Z_{a}\left(x, t \mid s, t_{0}\right)=0,
\end{aligned}
$$

to solve with the initial condition

$$
\lim _{t_{0} \uparrow t} Z_{a}\left(x, t \mid s, t_{0}\right)=e^{-s x},
$$

derived from (9) by using the initial condition (4).

Proposition 1 We assume that $\alpha(t) \in \mathbb{R}, \beta(t) \in \mathbb{R}, r(t)>0, \beta(t) \leq \xi r(t)$, with $0 \leq \xi<1$. For $t \geq t_{0}$, we have:

$$
\begin{gathered}
Z_{a}\left(x, t \mid s, t_{0}\right)=\frac{e^{-A\left(t \mid t_{0}\right)}}{\left[1+s R\left(t \mid t_{0}\right)\right]^{2}} \exp \left\{-\frac{s x e^{-A\left(t \mid t_{0}\right)}}{1+s R\left(t \mid t_{0}\right)}\right\} \\
\quad \times \exp \left\{\int_{t_{0}}^{t} \beta(u) \frac{s e^{-A\left(u \mid t_{0}\right)}}{1+s R\left(u \mid t_{0}\right)} d u\right\}, \quad x \geq 0,
\end{gathered}
$$

where

$$
A\left(t \mid t_{0}\right)=\int_{t_{0}}^{t} \alpha(z) d z, \quad R\left(t \mid t_{0}\right)=\int_{t_{0}}^{t} r(\tau) e^{-A\left(\tau \mid t_{0}\right)} d \tau .
$$

Proof The proof is given in Appendix A. 


\subsection{Proportional Case}

For all $t \geq 0$, we suppose that the continuous functions $\beta(t)$ and $r(t)$ are proportional, i.e.

$$
\frac{\beta(t)}{r(t)}=\xi, \quad 0 \leq \xi<1 .
$$

In the absence of immigration, i.e. when $\beta(t)=0$ for all $t \geq 0$, one has $\xi=0$.

Proposition 2 Under the assumption (14), for $t \geq t_{0}$ one has:

$$
Z_{a}\left(x, t \mid s, t_{0}\right)=\frac{e^{-A\left(t \mid t_{0}\right)}}{\left[1+s R\left(t \mid t_{0}\right)\right]^{2-\xi}} \exp \left\{-\frac{s x e^{-A\left(t \mid t_{0}\right)}}{1+s R\left(t \mid t_{0}\right)}\right\}, \quad x \geq 0 .
$$

Furthermore, the transition pdf of $X(t)$ in the presence of an absorbing boundary in the zero-state is:

$$
f_{a}\left(x, t \mid x_{0}, t_{0}\right)= \begin{cases}\frac{e^{-A\left(t \mid t_{0}\right)}}{\Gamma(2-\xi)}\left[\frac{1}{R\left(t \mid t_{0}\right)}\right]^{2-\xi} x_{0}^{1-\xi} \exp \left\{-\frac{x_{0}}{R\left(t \mid t_{0}\right)}\right\}, & x=0, \\ \frac{e^{-A\left(t \mid t_{0}\right)}}{R\left(t \mid t_{0}\right)}\left(\frac{x_{0}}{x}\right)^{(1-\xi) / 2} \exp \left\{-\frac{x_{0}+x e^{-A\left(t \mid t_{0}\right)}}{R\left(t \mid t_{0}\right)}\right\} \\ \quad \times \exp \left\{\frac{1-\xi}{2} A\left(t \mid t_{0}\right)\right\} I_{1-\xi}\left[\frac{2 \sqrt{x x_{0} e^{-A\left(t \mid t_{0}\right)}}}{R\left(t \mid t_{0}\right)}\right], & x>0,\end{cases}
$$

with $A\left(t \mid t_{0}\right)$ and $R\left(t \mid t_{0}\right)$ defined in (13) and where

$$
I_{\nu}(z)=\sum_{k=0}^{+\infty} \frac{1}{k ! \Gamma(v+k+1)}\left(\frac{z}{2}\right)^{2 k+v}, \quad v \in \mathbb{R}
$$

denotes the modified Bessel function of the first kind.

Proof The proof is given in Appendix B.

Note that, the first of (16) follows by taking the limit as $x \downarrow 0$ in the second, recalling that for fixed $v$ and for $z \rightarrow 0$ one has (cf. Abramowitz and Stegun [42], p. 375, no 9.6.7):

$$
I_{v}(z) \sim \frac{1}{\Gamma(v+1)}\left(\frac{z}{2}\right)^{v} \quad v \neq-1,-2, \ldots
$$

If (14) holds, for $t \geq t_{0}, x>0$ and $x_{0}>0$ from (16) it follows:

$$
f_{a}\left(x, t \mid x_{0}, t_{0}\right)=\left(\frac{x_{0}}{x}\right)^{1-\xi} \exp \left\{\frac{\left(x-x_{0}\right)\left[1-e^{-A\left(t \mid t_{0}\right)}\right]}{R\left(t \mid t_{0}\right)}\right\} f_{a}\left(x_{0}, t \mid x, t_{0}\right) .
$$

Proposition 3 Under the assumption (14), for $t \geq t_{0}$ and $x_{0}>0$ one has:

$$
\int_{0}^{+\infty} f_{a}\left(x, t \mid x_{0}, t_{0}\right) d x=\frac{1}{\Gamma(1-\xi)} \gamma\left(1-\xi, \frac{x_{0}}{R\left(t \mid t_{0}\right)}\right), \quad 0 \leq \xi<1,
$$

with $R\left(t \mid t_{0}\right)$ given in (13) and where

$$
\gamma(a, z)=\int_{0}^{z} e^{-y} y^{a-1} d y, \quad \operatorname{Re} a>0
$$

denotes the incomplete gamma function. 
Proof Recalling (16) and using the transformation $y=x e^{-A\left(t \mid t_{0}\right)} / R\left(t \mid t_{0}\right)$ in the integral, one obtains:

$$
\begin{aligned}
& \int_{0}^{+\infty} f_{a}\left(x, t \mid x_{0}, t_{0}\right) d x=\exp \left\{-\frac{x_{0}}{R\left(t \mid t_{0}\right)}\right\}\left[\frac{x_{0}}{R\left(t \mid t_{0}\right)}\right]^{(1-\xi) / 2} \\
& \quad \times \int_{0}^{+\infty} e^{-y} y^{-(1-\xi) / 2} I_{1-\xi}\left[2 \sqrt{\frac{x_{0} y}{R\left(t \mid t_{0}\right)}}\right] d y, \quad 0 \leq \xi<1 .
\end{aligned}
$$

Since (cf. Erdèlyi et al. [43], p. 197, no. 19)

$$
\int_{0}^{+\infty} e^{-p y} y^{-v / 2} I_{\nu}(2 \sqrt{a y}) d y=a^{-v / 2} p^{\nu-1} e^{a / p} \frac{\gamma(v, a / p)}{\Gamma(v)}, \quad \operatorname{Re} p>0,
$$

Eq. (19) follows from (21).

Proposition 4 Under the assumption (14), for $t \geq t_{0}$ and $x_{0}>0$ the FPT pdf through the zero-state of $X(t)$ is:

$$
g\left(0, t \mid x_{0}, t_{0}\right)=\frac{1}{\Gamma(1-\xi)} \frac{r(t) e^{-A\left(t \mid t_{0}\right)}}{R\left(t \mid t_{0}\right)}\left[\frac{x_{0}}{R\left(t \mid t_{0}\right)}\right]^{1-\xi} \exp \left\{-\frac{x_{0}}{R\left(t \mid t_{0}\right)}\right\}
$$

with $R\left(t \mid t_{0}\right)$ given in (13). Furthermore, for $0 \leq \xi<1$ the ultimate FPT probability is:

$$
P\left\{T\left(x_{0}, t_{0}\right)<+\infty\right\}= \begin{cases}1, & \lim _{t \rightarrow+\infty} R\left(t \mid t_{0}\right)=+\infty, \\ 1-\frac{\gamma\left(1-\xi, x_{0} / c\right)}{\Gamma(1-\xi)}, & \lim _{t \rightarrow+\infty} R\left(t \mid t_{0}\right)=c<+\infty .\end{cases}
$$

Proof By virtue of (8), making use of (19), for $0 \leq \xi<1$ one has:

$$
g\left(0, t \mid x_{0}, t_{0}\right)=-\frac{\partial}{\partial t} \int_{0}^{+\infty} f_{a}\left(x, t \mid x_{0}, t_{0}\right) d x=-\frac{1}{\Gamma(1-\xi)} \frac{\partial}{\partial t} \gamma\left(1-\xi, \frac{x_{0}}{R\left(t \mid t_{0}\right)}\right),
$$

from which (22) follows. Furthermore, taking the limit as $t \rightarrow+\infty$ in (8), it results

$$
P\left\{T\left(x_{0}, t_{0}\right)<+\infty\right\}=\int_{0}^{+\infty} g\left(0, t \mid x_{0}, t_{0}\right) d t=1-\lim _{t \rightarrow+\infty} \int_{0}^{+\infty} f_{a}\left(x, t \mid x_{0}, t_{0}\right) d x,
$$

so that, recalling (19), one is lead to (23).

Two interesting cases occur when $\xi=0$ and $\xi=1 / 2$.

Indeed, by setting $\xi=0$ in (14), one considers the time-inhomogeneous Feller-type diffusion process (1) with $\beta(t)=0$. In the context of population dynamics, this case describes the absence of the immigration and it is of interest to determine for which choices of $\alpha(t)$ and $r(t)$ the population is doomed to extinction as the time increases. Recalling that $\gamma(1, z)=$ $1-e^{-z}$, for $t \geq t_{0}, x_{0}>0$ and $\xi=0$, from (19) one has

$$
\int_{0}^{+\infty} f_{a}\left(x, t \mid x_{0}, t_{0}\right) d x=1-\exp \left\{-\frac{x_{0}}{R\left(t \mid t_{0}\right)}\right\}
$$

and from (22) one obtains:

$$
g\left(0, t \mid x_{0}, t_{0}\right)=\frac{r(t) x_{0} e^{-A\left(t \mid t_{0}\right)}}{R^{2}\left(t \mid t_{0}\right)} \exp \left\{-\frac{x_{0}}{R\left(t \mid t_{0}\right)}\right\} .
$$


Instead, when $\xi=1 / 2$ in (14), the FPT pdf (22) identifies with the FPT pdf through the zero-state for special time-inhomogeneous Wiener or Ornstein-Uhlenbeck diffusion processes (see, for instance, Giorno and Nobile [1]). Specifically, if $\xi=1 / 2$ and $\alpha(t)=0$, the density (22) identifies with the FPT pdf $g_{W}\left(0, t \mid \sqrt{x_{0}}, t_{0}\right)$ of a time-inhomogeneous Wiener process, with state-space in $\mathbb{R}$, having infinitesimal drift $B_{1}(x, t)=0$ and infinitesimal variance $B_{2}(t)=r(t) / 2$; instead, if $\xi=1 / 2$ and $\alpha(t) \neq 0$, the density (22) identifies with the FPT pdf $g_{O U}\left(0, t \mid \sqrt{x_{0}}, t_{0}\right)$ of a time-inhomogeneous Ornstein-Uhlenbeck process, with state-space in $\mathbb{R}$, having infinitesimal drift $C_{1}(x, t)=[\alpha(t) / 2] x$ and infinitesimal variance $C_{2}(t)=r(t) / 2$.

Under the assumption (14), if $\lim _{t \rightarrow+\infty} R\left(t \mid t_{0}\right)=+\infty$, it is meaningful to evaluate the FPT moments through the zero-state starting from $X\left(t_{0}\right)=x_{0}>0$ :

$$
t_{k}\left(0 \mid x_{0}, t_{0}\right)=\int_{0}^{+\infty} t^{k} g\left(0, t \mid x_{0}, t_{0}\right) d t, \quad k=1,2, \ldots
$$

Indeed, if $\lim _{t \rightarrow+\infty} R\left(t \mid t_{0}\right)=+\infty$, from Proposition 4 one has $P\left\{T\left(x_{0}, t_{0}\right)<+\infty\right\}=1$ and, making use of (8) and (19), for $0 \leq \xi<1$ one has:

$$
\begin{aligned}
& t_{k}\left(0 \mid x_{0}, t_{0}\right)=k \int_{0}^{+\infty} t^{k-1}\left[\int_{0}^{+\infty} f_{a}\left(x, t \mid x_{0}, t_{0}\right) d x\right] d t \\
& =\frac{k}{\Gamma(1-\xi)} \int_{0}^{+\infty} t^{k-1} \gamma\left(1-\xi, \frac{x_{0}}{R\left(t \mid t_{0}\right)}\right) d t, \quad k=1,2, \ldots
\end{aligned}
$$

We finally note that, for the time-homogeneous Feller process, in which $\alpha(t)=\alpha, \beta(t)=$ $\xi r, r(t)=r$, with $\alpha \in \mathbb{R}, r>0$ and $0 \leq \xi<1$, the pdf $f_{a}\left(x, t \mid x_{0}, t_{0}\right)$ and the FPT pdf $g\left(0, t \mid x_{0}, t_{0}\right)$ can be easily obtained from (16) and (22) by setting

$$
A\left(t \mid t_{0}\right)=\alpha\left(t-t_{0}\right), \quad R\left(t \mid t_{0}\right)= \begin{cases}r\left(t-t_{0}\right), & \alpha=0, \\ \frac{r}{\alpha}\left(1-e^{-\alpha\left(t-t_{0}\right)}\right), & \alpha \neq 0 .\end{cases}
$$

\section{General Case}

We assume that $\alpha(t), \beta(t)$ and $r(t)$ are continuous functions such that $\alpha(t) \in \mathbb{R}, \beta(t) \in \mathbb{R}$, $r(t)>0, \beta(t) \leq \xi r(t)$, with $0 \leq \xi<1$. From (12), for $t \geq t_{0}$ we have

$$
Z_{a}\left(x, t \mid s, t_{0}\right)= \begin{cases}Z_{a}\left(0, t \mid s, t_{0}\right), & x=0, \\ Z_{a}\left(0, t \mid s, t_{0}\right) V_{a}\left(x, t \mid s, t_{0}\right), & x>0,\end{cases}
$$

where

$$
V_{a}\left(x, t \mid s, t_{0}\right)=\exp \left\{-\frac{s x e^{-A\left(t \mid t_{0}\right)}}{1+s R\left(t \mid t_{0}\right)}\right\}
$$

with $A\left(t \mid t_{0}\right)$ and $R\left(t \mid t_{0}\right)$ given in (13). We note that $V_{a}\left(x, t \mid s, t_{0}\right)$ does not dependent upon $\beta(t)$. Therefore, to obtain the transition pdf $f_{a}\left(x, t \mid x_{0}, t_{0}\right)$ for $X(t)$ with infinitesimal moments (1), we proceed as follows:

(1) we determine the transition pdf $f_{a}\left(0, t \mid x_{0}, t_{0}\right)$ for $x_{0}>0$ and $t \geq t_{0}$ by taking the inverse Laplace transform of $Z_{a}\left(0, t \mid s, t_{0}\right)$; 
(2) we find the inverse Laplace transform $v_{a}\left(x, t \mid x_{0}, t_{0}\right)$ of (29) and we calculate the transition pdf $f_{a}\left(x, t \mid x_{0}, t_{0}\right)$ as a convolution, according to $x_{0}$, between $f_{a}\left(0, t \mid x_{0}, t_{0}\right)$ and the function $v_{a}\left(x, t \mid x_{0}, t_{0}\right)$ for $x>0, x_{0}>0$ and $t \geq t_{0}$.

\subsection{General Case: $x=0$}

In this section, we obtain the transition pdf in the presence of an absorbing boundary in the zero-state when the process $X(t)$ reaches $x=0$ at time $t \geq t_{0}$. By setting $x=0$ in (12), for $t \geq t_{0}$ we obtain:

$$
Z_{a}\left(0, t \mid s, t_{0}\right)=\frac{e^{-A\left(t \mid t_{0}\right)}}{\left[1+s R\left(t \mid t_{0}\right)\right]^{2}} \exp \left\{\int_{t_{0}}^{t} \beta(u) \frac{s e^{-A\left(u \mid t_{0}\right)}}{1+s R\left(u \mid t_{0}\right)} d u\right\},
$$

with $A\left(t \mid t_{0}\right)$ and $R\left(t \mid t_{0}\right)$ defined in (13).

In the sequel, we denote by $B_{n}\left(d_{1}, d_{2}, \ldots, d_{n}\right)$ the complete Bell polynomials, recursively defined as follows:

$$
B_{0}=1, \quad B_{n+1}\left(d_{1}, d_{2}, \ldots, d_{n+1}\right)=\sum_{i=0}^{n}\left(\begin{array}{l}
n \\
i
\end{array}\right) B_{n-i}\left(d_{1}, d_{2}, \ldots, d_{n-i}\right) d_{i+1}, \quad n \in \mathbb{N}_{0},
$$

with

$$
d_{k}=\frac{k !}{\left[R\left(t \mid t_{0}\right)\right]^{k}} \int_{t_{0}}^{t} \beta(u) e^{-A\left(u \mid t_{0}\right)}\left[R\left(t \mid t_{0}\right)-R\left(u \mid t_{0}\right)\right]^{k-1} d u, \quad k=1,2, \ldots
$$

Proposition 5 Under the assumption of Proposition 1 , for $t \geq t_{0}$ and $x_{0}>0$ the transition $p d f$ of the time-inhomogeneous Feller-type diffusion process $X(t)$ with an absorbing boundary in the zero-state is

$$
f_{a}\left(0, t \mid x_{0}, t_{0}\right)=\frac{x_{0} e^{-A\left(t \mid t_{0}\right)}}{R^{2}\left(t \mid t_{0}\right)} \exp \left\{-\frac{x_{0}}{R\left(t \mid t_{0}\right)}\right\} \Psi\left(t \mid x_{0}, t_{0}\right)
$$

where

$$
\Psi\left(t \mid z, t_{0}\right)=\sum_{n=0}^{+\infty} \frac{B_{n}\left(d_{1}, d_{2}, \ldots, d_{n}\right)}{(n+1) !} L_{n}^{(1)}\left[\frac{z}{R\left(t \mid t_{0}\right)}\right], \quad z>0,
$$

with $A\left(t \mid t_{0}\right)$ and $R\left(t \mid t_{0}\right)$ defined in (13), $B_{n}\left(d_{1}, d_{2}, \ldots, d_{n}\right)$ given in (31) and in (32), and

$$
L_{n}^{(a)}(y)=\sum_{k=0}^{n}(-1)^{k}\left(\begin{array}{l}
n+a \\
n-k
\end{array}\right) \frac{y^{k}}{k !}, \quad a \geq 0, n=0,1, \ldots
$$

denoting the Laguerre polynomials.

Proof The proof is given in Appendix C.

Remark 1 (Proportional case) We assume that (14) holds. We prove that the first of (16) follows from (33).

Indeed, from (31) and (32) one has

$$
d_{n}=\xi(n-1) !, \quad B_{0}=1, \quad B_{n}\left(d_{1}, d_{2}, \ldots, d_{n}\right)=(\xi)_{n}, \quad n=1,2, \ldots,
$$


where $(\xi)_{n}$ denotes the Pochhammer symbol defined as $(\xi)_{0}=1$ and $(\xi)_{n}=\xi(\xi+1) \cdots(\xi+$ $n-1)$ for $n=1,2, \ldots$ Recalling that the series of Laguerre polynomials satisfies the following identity (cf. Erdèlyi et al [44], p. 213, no. 16):

$$
\sum_{n=0}^{+\infty} \frac{(\xi)_{n}}{\Gamma(a+n+1)} L_{n}^{(a)}(y)=\frac{y^{-\xi}}{\Gamma(a-\xi+1)}, \quad a>0, y>0,0 \leq \xi<a+1,
$$

under the assumption (14), from (34) one has:

$$
\Psi\left(t \mid z, t_{0}\right)=\sum_{n=0}^{+\infty} \frac{(\xi)_{n}}{(n+1) !} L_{n}^{(1)}\left[\frac{z}{R\left(t \mid t_{0}\right)}\right]=\frac{1}{\Gamma(2-\xi)}\left[\frac{z}{R\left(t \mid t_{0}\right)}\right]^{-\xi}, \quad z>0 .
$$

Hence, if (14) holds, Eq. (33) identifies with the first of (16).

\subsection{General Case: $x>0$}

In this section, we obtain the transition pdf $f_{a}\left(x, t \mid x_{0}, t_{0}\right)$ for $x>0$ and $t \geq t_{0}$. From (29), for $t \geq t_{0}, x>0$ and $\operatorname{Re} s>0$ we have $V_{a}\left(x, t \mid s, t_{0}\right) \geq 0$ and

$$
\lim _{s \downarrow 0} V_{a}\left(x, t \mid s, t_{0}\right)=1 .
$$

We show that $V_{a}\left(x, t \mid s, t_{0}\right)$ is the Laplace transform of a function $v_{a}\left(x, t \mid x_{0}, t_{0}\right)$, i.e.

$$
V_{a}\left(x, t \mid s, t_{0}\right)=\int_{0}^{+\infty} e^{-s x_{0}} v_{a}\left(x, t \mid x_{0}, t_{0}\right) d x_{0}, \quad \operatorname{Re} s>0 .
$$

Proposition 6 Under the assumption of Proposition 1 , for $x_{0}>0$ and $t \geq t_{0}$, one has:

$$
\begin{aligned}
& v_{a}\left(x, t \mid x_{0}, t_{0}\right)=\exp \left\{-\frac{x e^{-A\left(t \mid t_{0}\right)}}{R\left(t \mid t_{0}\right)}\right\} \delta\left(x_{0}\right)+\frac{1}{R\left(t \mid t_{0}\right)} \sqrt{\frac{x}{x_{0}} e^{-A\left(t \mid t_{0}\right)}} \\
& \times \exp \left\{-\frac{x_{0}+x e^{-A\left(t \mid t_{0}\right)}}{R\left(t \mid t_{0}\right)}\right\} I_{1}\left[\frac{2 \sqrt{x x_{0} e^{-A\left(t \mid t_{0}\right)}}}{R\left(t \mid t_{0}\right)}\right], \quad x>0,
\end{aligned}
$$

with $A\left(t \mid t_{0}\right)$ and $R\left(t \mid t_{0}\right)$ defined in (13), whereas $\delta(x)$ denotes the delta Dirac function and $I_{\nu}(z)$ represents the Bessel function modified of first kind.

Proof The proof is given in Appendix D.

The function $v_{a}\left(x, t \mid x_{0}, t_{0}\right)$ in (40) is the sum of two terms. The second term in (40) identifies with $x f_{a}\left(x, t \mid x_{0}, t_{0}\right) / x_{0}$, where $f_{a}\left(x, t \mid x_{0}, t_{0}\right)$ is given in (16) for $x>0$ and $\xi=0$ (absence of immigration). Since,

$$
\int_{0}^{+\infty} \frac{x}{x_{0}} f_{a}\left(x, t \mid x_{0}, t_{0}\right) d x_{0}=1-\exp \left\{-\frac{x e^{-A\left(t \mid t_{0}\right)}}{R\left(t \mid t_{0}\right)}\right\}, \quad x>0,
$$

from (40) it follows that

$$
\int_{0}^{+\infty} v_{a}\left(x, t \mid x_{0}, t_{0}\right) d x_{0}=1
$$

For $x>0$, the transition pdf $f_{a}\left(x, t \mid x_{0}, t_{0}\right)$ can be obtained via a convolution, according to $x_{0}$, between the pdf $f_{a}\left(0, t \mid x_{0}, t_{0}\right)$ and the function $v_{a}\left(x, t \mid x_{0}, t_{0}\right)$, determined in Propositions 5 
and 6, respectively:

$$
f_{a}\left(x, t \mid x_{0}, t_{0}\right)=\int_{0}^{x_{0}} f_{a}\left(0, t \mid z, t_{0}\right) v_{a}\left(x, t \mid x_{0}-z, t_{0}\right) d z, \quad x>0, x_{0}>0 .
$$

Proposition 7 Under the assumption of Proposition 1, for $t \geq t_{0}, x>0$ and $x_{0}>0$ one has:

$$
\begin{aligned}
& f_{a}\left(x, t \mid x_{0}, t_{0}\right)=\frac{e^{-A\left(t \mid t_{0}\right)}}{R^{2}\left(t \mid t_{0}\right)} \exp \left\{-\frac{x_{0}+x e^{-A\left(t \mid t_{0}\right)}}{R\left(t \mid t_{0}\right)}\right\}\left\{x_{0} \Psi\left(t \mid x_{0}, t_{0}\right)\right. \\
& \left.+\frac{\sqrt{x e^{-A\left(t \mid t_{0}\right)}}}{R\left(t \mid t_{0}\right)} \int_{0}^{x_{0}} \frac{z}{\sqrt{x_{0}-z}} I_{1}\left[\frac{2 \sqrt{x\left(x_{0}-z\right) e^{-A\left(t \mid t_{0}\right)}}}{R\left(t \mid t_{0}\right)}\right] \Psi\left(t \mid z, t_{0}\right) d z\right\},
\end{aligned}
$$

with $A\left(t \mid t_{0}\right)$ and $R\left(t \mid t_{0}\right)$ given in (13) and $\Psi\left(t \mid z, t_{0}\right)$ defined in (34).

Proof It follows from (41), by virtue of (33) and (40).

Note that, by taking the limit as $x \downarrow 0$ in (42), we obtain (33).

Remark 2 (Proportional case) We assume that (14) holds. We prove that the second of (16) follows from (42).

Indeed, recalling (36) and (38), from (42) for $t \geq t_{0}, x>0$ and $x_{0}>0$ one has:

$$
\begin{aligned}
& f_{a}\left(x, t \mid x_{0}, t_{0}\right)=\frac{e^{-A\left(t \mid t_{0}\right)}}{\Gamma(2-\xi)}\left[\frac{1}{R\left(t \mid t_{0}\right)}\right]^{2-\xi} \exp \left\{-\frac{x_{0}+x e^{-A\left(t \mid t_{0}\right)}}{R\left(t \mid t_{0}\right)}\right\} \\
& \quad \times\left\{x_{0}^{1-\xi}+\frac{\sqrt{x e^{-A\left(t \mid t_{0}\right)}}}{R\left(t \mid t_{0}\right)} \int_{0}^{x_{0}} \frac{\left(x_{0}-y\right)^{1-\xi}}{\sqrt{y}} I_{1}\left[\frac{2 \sqrt{x y e^{-A\left(t \mid t_{0}\right)}}}{R\left(t \mid t_{0}\right)}\right] d y\right\} .
\end{aligned}
$$

By virtue of (17), one obtains

$$
\int_{0}^{x_{0}} \frac{\left(x_{0}-y\right)^{1-\xi}}{\sqrt{y}} I_{1}(2 a \sqrt{y}) d y=\frac{x_{0}^{-\xi}}{a^{2}}\left\{-a x_{0}+a^{\xi} x_{0}^{(1+\xi) / 2} I_{1-\xi}\left(2 a \sqrt{x_{0}}\right) \Gamma(2-\xi)\right\},
$$

for $a>0, \xi<2$ and $x_{0}>0$. Hence, under the assumption (14), Eq. (43) leads to the second of (16).

\section{The First-Passage Time Through the Zero-State}

We now focus on the distribution function of the FPT through the zero-state for the timeinhomogeneous Feller-type diffusion process $X(t)$, with infinitesimal moments (1), when $\alpha(t), \beta(t)$ and $r(t)$ are continuous functions such that $\alpha(t) \in \mathbb{R}, \beta(t) \in \mathbb{R}, r(t)>0$, $\beta(t) \leq \xi r(t)$, with $0 \leq \xi<1$. The FPT problem of $X(t)$ through the zero-state can be studied starting from Eq. (8) and making use of (42).

Proposition 8 Under the assumption of Proposition 1 , for $t \geq t_{0}$ and $x_{0}>0$ one has:

$$
\begin{aligned}
& \int_{0}^{+\infty} f_{a}\left(x, t \mid x_{0}, t_{0}\right) d x=1-\exp \left\{-\frac{x_{0}}{R\left(t \mid t_{0}\right)}\right\}+\frac{x_{0}}{R\left(t \mid t_{0}\right)} \exp \left\{-\frac{x_{0}}{R\left(t \mid t_{0}\right)}\right\} \\
& \quad \times \sum_{n=1}^{+\infty} \frac{B_{n}\left(d_{1}, d_{2}, \ldots, d_{n}\right)}{n !} \Phi\left(1-n, 2 ; \frac{x_{0}}{R\left(t \mid t_{0}\right)}\right),
\end{aligned}
$$


with $R\left(t \mid t_{0}\right)$ defined in (13), $B_{n}\left(d_{1}, d_{2}, \ldots, d_{n}\right)$ given in (31) and (32) and where

$$
\Phi(a, b ; x)=1+\sum_{n=1}^{+\infty} \frac{(a)_{n}}{(b)_{n}} \frac{x^{n}}{n !}
$$

denotes the confluent hypergeometric function (Kummer's function).

Proof The proof is given in Appendix E.

Remark 3 (Proportional case) We assume that (14) holds. We prove that from (44) one obtains (19).

Indeed, recalling (36) and making use of the relation $\Phi(1,2 ; z)=\left(e^{z}-1\right) / z$, from (44) for $t \geq t_{0}$ and $x_{0}>0$ one has:

$$
\int_{0}^{+\infty} f_{a}\left(x, t \mid x_{0}, t_{0}\right) d x=\frac{x_{0}}{R\left(t \mid t_{0}\right)} \exp \left\{-\frac{x_{0}}{R\left(t \mid t_{0}\right)}\right\} \sum_{n=0}^{+\infty} \frac{(\xi)_{n}}{n !} \Phi\left(1-n, 2 ; \frac{x_{0}}{R\left(t \mid t_{0}\right)}\right) .
$$

Since (Tricomi [45], p. 31, no. 10)

$$
\sum_{n=0}^{+\infty} \frac{(b-c)_{n}}{n !} \Phi(a-n, b ; z)=\frac{\Gamma(b)}{\Gamma(c)} z^{c-b} \Phi(a, c ; z), \quad b>0, c>0, b-c>0,
$$

from (46) one has:

$$
\int_{0}^{+\infty} f_{a}\left(x, t \mid x_{0}, t_{0}\right) d x=\frac{e^{-x_{0} / R\left(t \mid t_{0}\right)}}{\Gamma(2-\xi)}\left[\frac{x_{0}}{R\left(t \mid t_{0}\right)}\right]^{1-\xi} \Phi\left(1,2-\xi ; \frac{x_{0}}{R\left(t \mid t_{0}\right)}\right) .
$$

The incomplete gamma function (20) can be expressed in terms of the Kummer's function (cf. Tricomi [45]p. 160, no. 7):

$$
\gamma(a, z)=\frac{1}{a} e^{-z} z^{a} \Phi(1, a+1, z), \quad \operatorname{Re} a>0
$$

so that Eq. (19) follows from (48).

Relation (44) plays an important role in the determination of the FPT distribution function and of the FPT density through the zero-state. Indeed, by virtue of (8), for $t \geq t_{0}$ and $x_{0}>0$ the FPT distribution function is

$$
P\left\{T\left(x_{0}, t_{0}\right)<t\right\}=\int_{t_{0}}^{t} g\left(0, \tau \mid x_{0}, t_{0}\right) d \tau=1-\int_{0}^{+\infty} f_{a}\left(x, t \mid x_{0}, t_{0}\right) d x,
$$

so that the FPT density through the zero-state can be obtained as

$$
g\left(0, t \mid x_{0}, t_{0}\right)=-\frac{\partial}{\partial t} \int_{0}^{+\infty} f_{a}\left(x, t \mid x_{0}, t_{0}\right) d x .
$$

Proposition 9 Under the assumption of Proposition 1 , for $t \geq t_{0}$ and $x_{0}>0$ one has:

$$
\begin{aligned}
& g\left(0, t \mid x_{0}, t_{0}\right)=\frac{x_{0}}{R\left(t \mid t_{0}\right)} \exp \left\{-\frac{x_{0}}{R\left(t \mid t_{0}\right)}\right\}\left\{\frac{r(t) e^{-A\left(t \mid t_{0}\right)}}{R\left(t \mid t_{0}\right)}\right. \\
& \quad \times \sum_{n=0}^{+\infty} \frac{B_{n}\left(d_{1}, d_{2}, \ldots, d_{n}\right)}{n !} \Phi\left(-n, 1 ; \frac{x_{0}}{R\left(t \mid t_{0}\right)}\right) \\
& \left.-\sum_{n=1}^{+\infty} \frac{1}{n !} \Phi\left(1-n, 2 ; \frac{x_{0}}{R\left(t \mid t_{0}\right)}\right) \frac{d}{d t} B_{n}\left(d_{1}, d_{2}, \ldots, d_{n}\right)\right\},
\end{aligned}
$$


with $A\left(t \mid t_{0}\right)$ and $R\left(t \mid t_{0}\right)$ defined in (13) and $B_{n}\left(d_{1}, d_{2}, \ldots, d_{n}\right)$ given in (31) and (32).

Proof The proof is given in Appendix F.

Remark 4 (Proportional case) We assume that (14) holds. We prove that from (51) one obtains (22).

Indeed, from (36) one has $B_{0}=1$ and $B_{n}\left(d_{1}, d_{2}, \ldots, d_{n}\right)=(\xi)_{n}$ for $n=1,2, \ldots$, so that, under the assumption (14), for $t \geq t_{0}$ and $x_{0}>0$ from (51) one has:

$$
g\left(0, t \mid x_{0}, t_{0}\right)=\frac{x_{0} r(t) e^{-A\left(t \mid t_{0}\right)}}{R^{2}\left(t \mid t_{0}\right)} \exp \left\{-\frac{x_{0}}{R\left(t \mid t_{0}\right)}\right\} \sum_{n=0}^{+\infty} \frac{(\xi)_{n}}{n !} \Phi\left(-n, 1 ; \frac{x_{0}}{R\left(t \mid t_{0}\right)}\right) .
$$

Making use of (47), it results

$$
\sum_{n=0}^{+\infty} \frac{(\xi)_{n}}{n !} \Phi\left(-n, 1 ; \frac{x_{0}}{R\left(t \mid t_{0}\right)}\right)=\frac{1}{\Gamma(1-\xi)}\left[\frac{x_{0}}{R\left(t \mid t_{0}\right)}\right]^{-\xi},
$$

so that (22) follows from (52).

\section{Special Cases}

Under the assumption (14), we analyze the cases in which the growth intensity function $\alpha(t)$, or the immigration intensity function $\beta(t)$ or both of them have some kind of periodicity. These cases are of interest in various applied fields, such as in population growth and in queueing systems. Indeed, periodic immigration intensity functions play an important role in the description of the evolution of dynamic for systems influenced by seasonal immigration or other regular environmental cycles. Furthermore, periodic growth intensity functions express the existence of fluctuation in the population dynamics and the presence of rush hours occurring on a daily basis in queueing systems.

\subsection{Periodic Immigration Intensity Function}

We consider the time-inhomogeneous Feller-type process $X(t)$ such that

$$
A_{1}(x, t)=\alpha x+\xi r(t), \quad A_{2}(x, t)=2 r(t) x,
$$

with $\alpha \in \mathbb{R}, 0 \leq \xi<1$ and

$$
r(t)=v\left[1+c \sin \left(\frac{2 \pi t}{Q}\right)\right], \quad t \geq 0,
$$

where $v>0$ is the average of the periodic function $r(t)$ of period $Q, c$ is the amplitude of the oscillations, with $0 \leq c<1$. From (13), for $t \geq t_{0}$ one has $A\left(t \mid t_{0}\right)=\alpha\left(t-t_{0}\right)$ and

$$
R\left(t \mid t_{0}\right)= \begin{cases}v\left(t-t_{0}\right)+\frac{c v Q}{2 \pi}\left[\cos \left(\frac{2 \pi t_{0}}{Q}\right)-\cos \left(\frac{2 \pi t}{Q}\right)\right], & \alpha=0, \\ \frac{v}{\alpha}\left(1-e^{-\alpha\left(t-t_{0}\right)}\right)+\frac{c v Q}{4 \pi^{2}+Q^{2} \alpha^{2}}\left\{2 \pi \cos \left(\frac{2 \pi t_{0}}{Q}\right)\right. & \\ \left.+\alpha Q \sin \left(\frac{2 \pi t_{0}}{Q}\right)-e^{-\alpha\left(t-t_{0}\right)}\left[2 \pi \cos \left(\frac{2 \pi t}{Q}\right)+\alpha Q \sin \left(\frac{2 \pi t}{Q}\right)\right]\right\}, \alpha \neq 0 .\end{cases}
$$



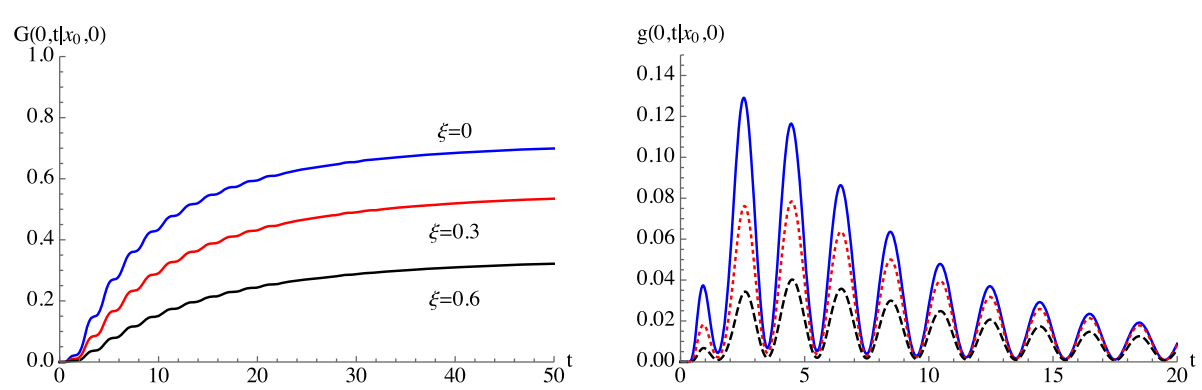

Fig. 1 The FPT distribution (on the left) and the FPT density (on the right) through the zero-state starting from $X(0)=5$ are plotted as function of $t$ for the process (53), with $\alpha=0.05, r(t)$ given in (54) with $v=0.75$, $c=0.9, Q=2$ with $\xi=0$ (blue solid curve), $\xi=0.3$ (red dotted curve) and $\xi=0.6$ (black dashed curve)
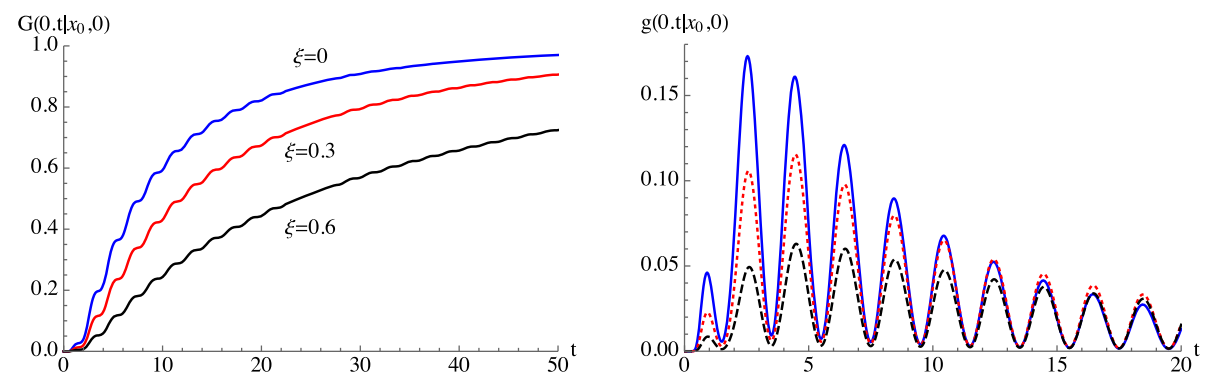

Fig. 2 As in Fig. 1 for $\alpha=-0.05$
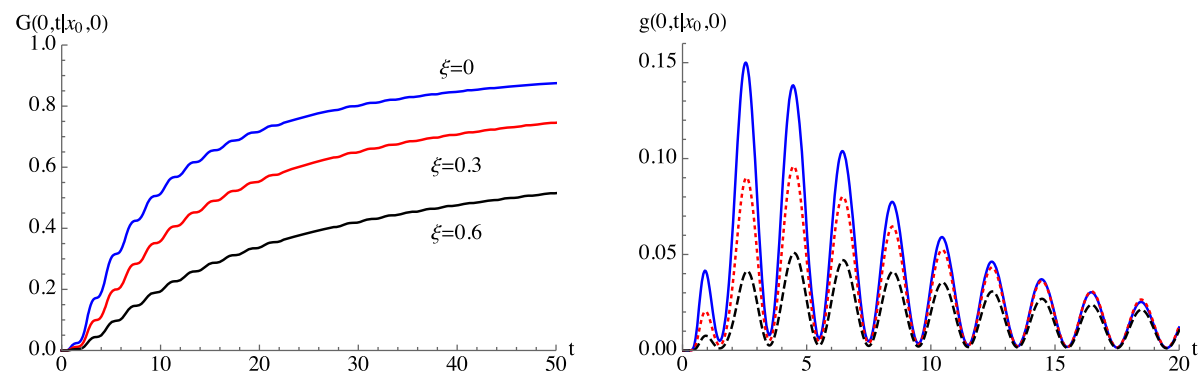

Fig. 3 As in Fig. 1 for $\alpha=0$

Then, from (55) one obtains:

$$
\lim _{t \rightarrow+\infty} R\left(t \mid t_{0}\right)= \begin{cases}+\infty, & \alpha \leq 0, \\ \frac{v}{\alpha}+\frac{c v Q}{4 \pi^{2}+Q^{2} \alpha^{2}}\left[2 \pi \cos \left(\frac{2 \pi t_{0}}{Q}\right)+\alpha Q \sin \left(\frac{2 \pi t_{0}}{Q}\right)\right], & \alpha>0,\end{cases}
$$

so that, by virtue of (23), the FPT through the zero-state is a certain event for $\alpha \leq 0$. Moreover, for $\alpha=0$ the FPT moments (26) are divergent.

In Figs. 1, 2 and 3, the FPT distribution $G\left(0, t \mid x_{0}, t_{0}\right)=1-\int_{0}^{+\infty} f_{a}\left(x, t \mid x_{0}, t_{0}\right) d x$, obtained making use of (19), and the FPT pdf $g\left(0, t \mid x_{0}, t_{0}\right)$, given in (22), are plotted as function of $t$ for the diffusion process (53) for some choices of parameters. In Fig. 4, the mean $t_{1}\left(0 \mid x_{0}, t_{0}\right)$ and the coefficient of variation $\mathrm{CV}\left(0 \mid x_{0}, t_{0}\right)$, obtained making use of (26), 

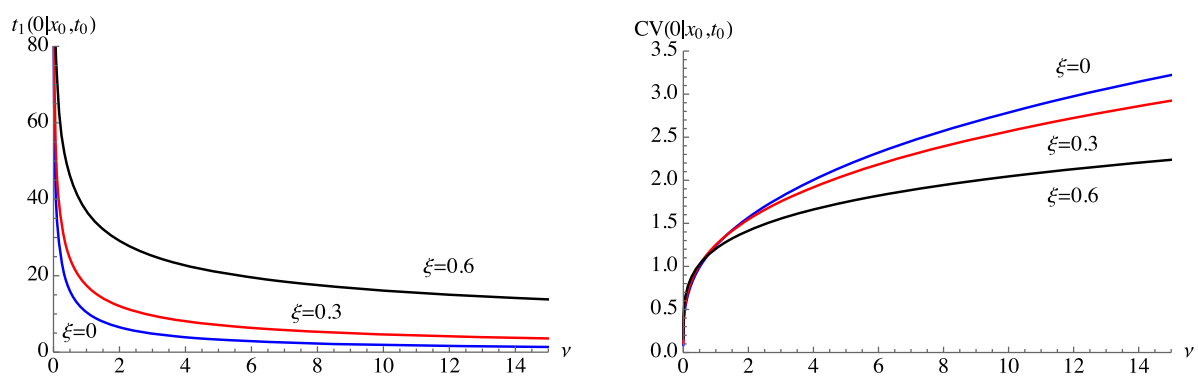

Fig. 4 The mean (on the left) and the coefficient of variation (on the right) of FPT from $X(0)=5$ through the zero-state are plotted as function of $v$ for the diffusion process (53) with $\alpha=-0.05, c=0.9, Q=2$

are plotted as function of $v$ for $\xi=0,0.3,0.6$. We note that as $v$ increases, the FPT mean $t_{1}\left(0 \mid x_{0}, t_{0}\right)$ decreases whereas the coefficient of variation increases. Instead, as $\xi$ increases in $[0,1)$, the FPT mean increases and the coefficient of variation decreases, due to a raise of the immigration intensity function.

\subsection{Periodic Growth Intensity Function}

We consider the time-inhomogeneous Feller-type process $X(t)$ such that

$$
A_{1}(x, t)=\alpha(t) x+\xi r, \quad A_{2}(x)=2 r x
$$

with $r>0,0 \leq \xi<1$ and

$$
\alpha(t)=\eta-\frac{2 \pi b}{Q_{1}} \frac{\cos \left(\frac{2 \pi t}{Q_{1}}\right)}{1+b \sin \left(\frac{2 \pi t}{Q_{1}}\right)}, \quad t \geq 0,
$$

where $\eta \in \mathbb{R}$ is the average of the periodic function $\alpha(t)$ of period $Q_{1}, b$ determines the amplitude of the oscillations, with $0 \leq b<1$. In Fig. 5, the intensity function (57) is plotted as function of $t$ for some choices of parameters $\eta, b$ and $Q_{1}$. The dotted lines refer to the average cases, in which $\alpha(t)=\eta$ with $\eta=-5$ (bottom) and $\eta=5$ (top). From (13), for $t \geq t_{0}$ one has

$$
A\left(t \mid t_{0}\right)=\eta\left(t-t_{0}\right)-\ln \left[1+b \sin \left(\frac{2 \pi t}{Q_{1}}\right)\right]+\ln \left[1+b \sin \left(\frac{2 \pi t_{0}}{Q_{1}}\right)\right],
$$

and

$$
R\left(t \mid t_{0}\right)=\left\{\begin{array}{l}
\frac{r}{1+b \sin \left(\frac{2 \pi t_{0}}{Q_{1}}\right)}\left\{t-t_{0}-\frac{b Q_{1}}{2 \pi}\left[\cos \left(\frac{2 \pi t}{Q_{1}}\right)-\cos \left(\frac{2 \pi t_{0}}{Q_{1}}\right)\right]\right\}, \quad \eta=0, \\
\frac{r}{1+b \sin \left(\frac{2 \pi t_{0}}{Q_{1}}\right)}\left\{\frac{1-e^{-\eta\left(t-t_{0}\right)}}{\eta}-\frac{2 \pi b Q_{1}}{4 \pi^{2}+Q_{1}^{2} \eta^{2}}\left[e^{-\eta\left(t-t_{0}\right)} \cos \left(\frac{2 \pi t}{Q_{1}}\right)\right.\right. \\
\left.+\frac{Q_{1} \eta}{2 \pi} e^{-\eta\left(t-t_{0}\right)} \sin \left(\frac{2 \pi t}{Q_{1}}\right)-\cos \left(\frac{2 \pi t_{0}}{Q_{1}}\right)-\frac{Q_{1} \eta}{2 \pi} \sin \left(\frac{2 \pi t_{0}}{Q_{1}}\right)\right\}, \quad \eta \neq 0 .
\end{array}\right.
$$



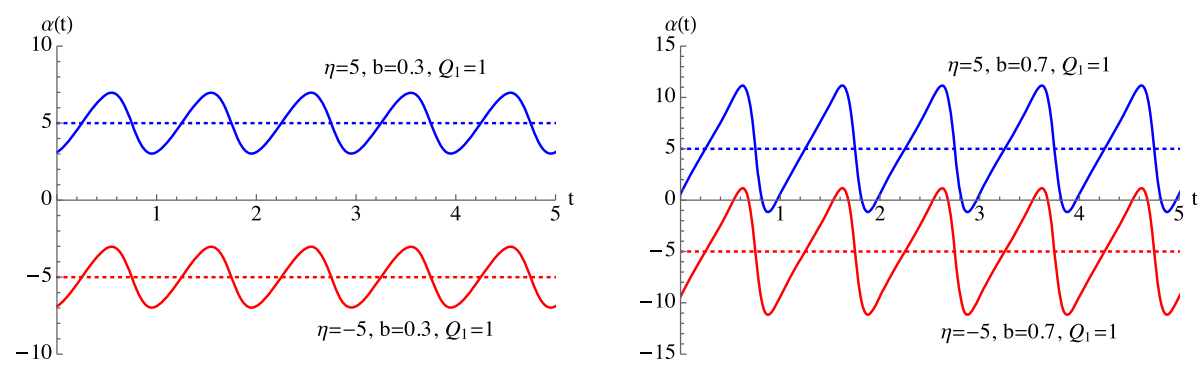

Fig. 5 The intensity function $\alpha(t)$, given in (57), is plotted as function of $t$ for some choices of parameters. The dotted lines refer to the average cases

Then, from (59) one obtains:

$$
\lim _{t \rightarrow+\infty} R\left(t \mid t_{0}\right)= \begin{cases}+\infty, & \eta \leq 0, \\ \frac{r\left\{\frac{1}{\eta}+\frac{2 \pi b Q_{1}}{4 \pi^{2}+Q_{1}^{2} \eta^{2}}\left[\cos \left(\frac{2 \pi t_{0}}{Q_{1}}\right)+\frac{Q_{1} \eta}{2 \pi} \sin \left(\frac{2 \pi t_{0}}{Q_{1}}\right)\right]\right\}}{1+b \sin \left(\frac{2 \pi t_{0}}{Q_{1}}\right)}, & \eta>0,\end{cases}
$$

so that, by virtue of (23), the FPT through zero-state is a certain event for $\eta \leq 0$. Moreover, for $\eta=0$ the FPT moments (26) are divergent.

In Fig. 6, the FPT pdf $g\left(0, t \mid x_{0}, t_{0}\right)$, given in (22), is plotted as function of $t$ for the process (56) for some choices of parameters. Instead, in Fig. 7, the mean $t_{1}\left(0 \mid x_{0}, t_{0}\right)$ and the coefficient of variation $\mathrm{CV}\left(0 \mid x_{0}, t_{0}\right)$, obtained making use of (26), are plotted as function of $r$ for $\xi=0,0.3,0.6$. We note that as $r$ increases, the FPT mean $t_{1}\left(0 \mid x_{0}, t_{0}\right)$ decreases, whereas the coefficient of variation increases. Moreover, the FPT mean and the coefficient of variation increase with $\xi$ in $[0,1)$.

\subsection{Periodic Immigration and Growth Intensity Functions}

We consider the time-inhomogeneous Feller-type process $X(t)$ such that

$$
A_{1}(x, t)=\alpha(t) x+\xi r(t), \quad A_{2}(x, t)=2 r(t) x,
$$

with $0 \leq \xi<1, r(t)$ defined in (54) and $\alpha(t)$ given in (57). Recalling (13), for $t \geq t_{0}$ one obtains $A\left(t \mid t_{0}\right)$ given in (58) and

$$
R\left(t \mid t_{0}\right)=\frac{v}{1+b \sin \left(\frac{2 \pi t_{0}}{Q_{1}}\right)} \int_{t_{0}}^{t} e^{-\eta\left(\tau-t_{0}\right)}\left[1+c \sin \left(\frac{2 \pi \tau}{Q}\right)\right]\left[1+b \sin \left(\frac{2 \pi \tau}{Q_{1}}\right)\right] d \tau
$$

The explicit expression of $R\left(t \mid t_{0}\right)$ in (61) is obtained in Appendix G. We note that $\lim _{t \rightarrow+\infty} R\left(t \mid t_{0}\right)$ diverges as $\eta \leq 0$, so that, due to (23), the FPT through the zero-state is a certain event for $X(t)$.

In Fig. 8, the FPT pdf $g\left(0, t \mid x_{0}, t_{0}\right)$, given in (22), is plotted as function of $t$ for the process (60) for some choices of parameters. Comparing Figs. 6 and 8 , we note the effect of the different periodicities of the growth intensity function $\alpha(t)$, with $Q_{1}=1$, and of the immigration intensity function $\beta(t)=\xi r(t)$, with $Q=2$. In Fig. 9, the mean $t_{1}\left(0 \mid x_{0}, t_{0}\right)$ and the coefficient of variation $\mathrm{CV}\left(0 \mid x_{0}, t_{0}\right)$, obtained making use of (26), are plotted as function 


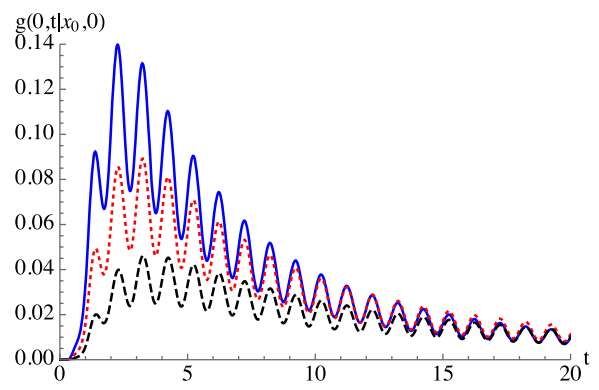

(a) $\eta=0, b=0.3, Q_{1}=1$

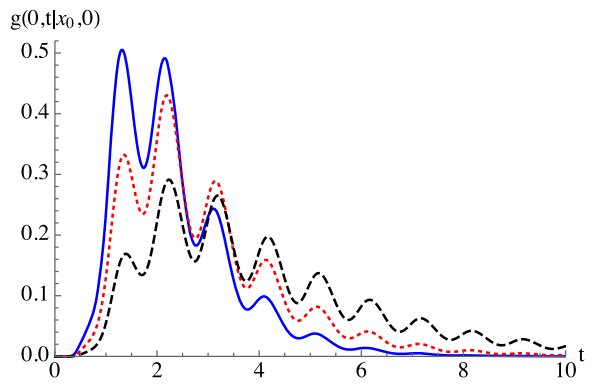

(c) $\eta=-1, b=0.3, Q_{1}=1$

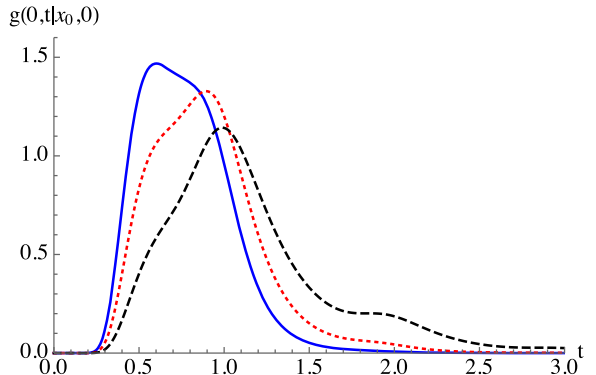

(e) $\eta=-5, b=0.3, Q_{1}=1$

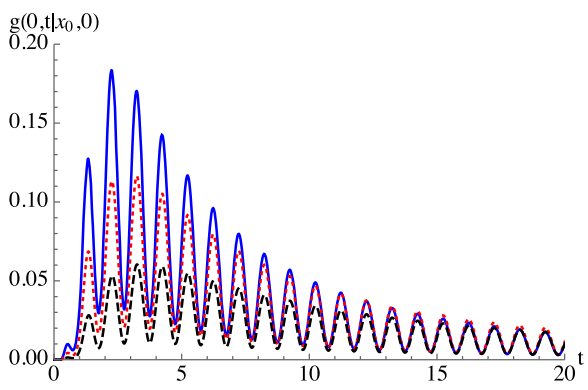

(b) $\eta=0, b=0.7, Q_{1}=1$

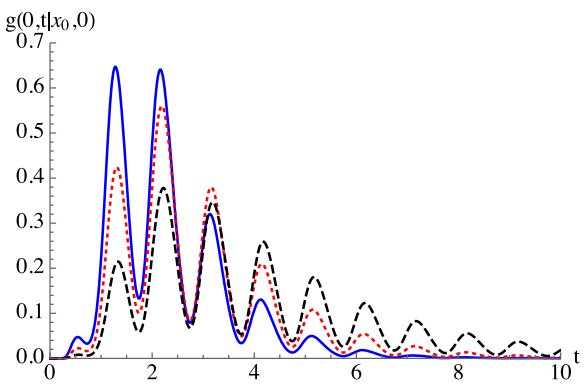

(d) $\eta=-1, b=0.7, Q_{1}=1$

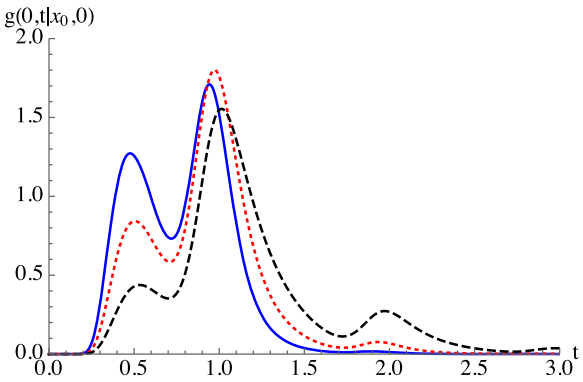

(f) $\eta=-5, b=0.7, Q_{1}=1$

Fig. 6 FPT densities through the zero-state starting from $X(0)=5$ are plotted as function of $t$ for the process (56), with $r=1, \alpha(t)$ given in (57) and with $\xi=0$ (blue solid curve), $\xi=0.3$ (red dotted curve) and $\xi=0.6$ (black dashed curve)
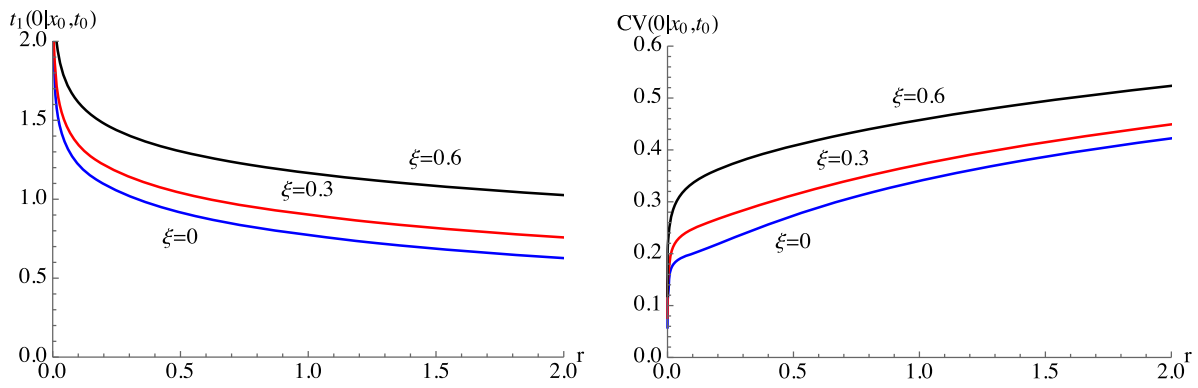

Fig. 7 The mean (on the left) and the coefficient of variation (on the right) of FPT from $X(0)=5$ to the zero-state are plotted as function of $r$ for the process (56) with $\eta=-5, b=0.3, Q_{1}=1$ 


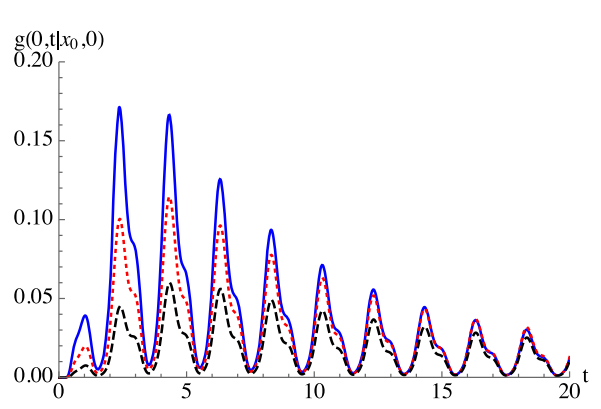

(a) $\eta=0, b=0.3, Q_{1}=1, Q=2$

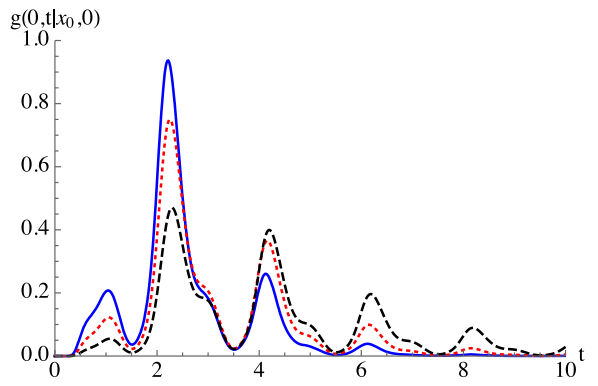

(c) $\eta=-1, b=0.3, Q_{1}=1, Q=2$

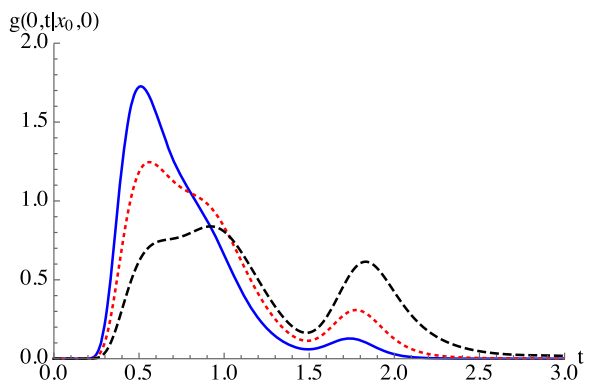

(e) $\eta=-5, b=0.3, Q_{1}=1, Q=2$

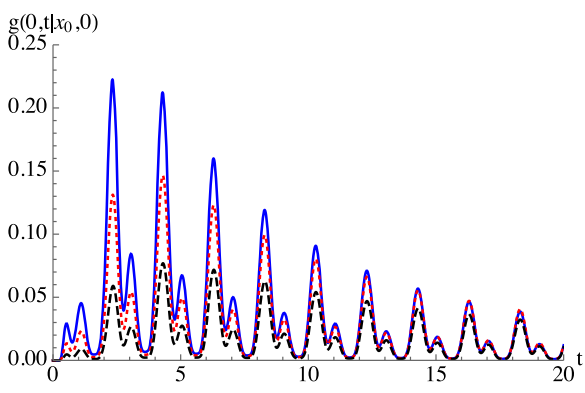

(b) $\eta=0, b=0.7, Q_{1}=1, Q=2$

$\mathrm{g}\left(0, \mathrm{t} \mid x_{0}, 0\right)$

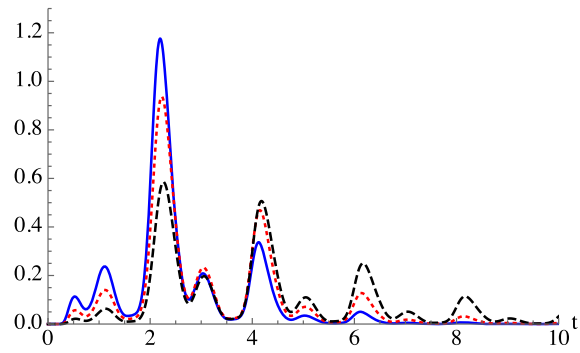

(d) $\eta=-1, b=0.7, Q_{1}=1, Q=2$

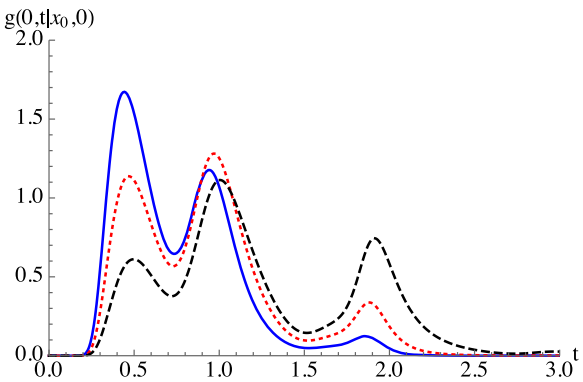

(f) $\eta=-5, b=0.7, Q_{1}=1, Q=2$

Fig. 8 FPT densities through the zero-state starting from $X(0)=5$ are plotted as function of $t$ for the process (60), being $r(t)$ defined in (54), with $v=0.75$ and $c=0.9$, and $\alpha(t)$ given in (57) with $\xi=0$ (blue solid curve), $\xi=0.3$ (red dotted curve) and $\xi=0.6$ (black dashed curve)

of $v$ for $\xi=0,0.3,0.6$. As $v$ increases, the FPT mean $t_{1}\left(0 \mid x_{0}, t_{0}\right)$ decreases whereas the coefficient of variation increases. Instead, as $\xi$ increases in $[0,1)$, both the FPT mean and the coefficient of variation increase.

\section{Concluding Remarks}

In this paper, we have considered a time-inhomogeneous Feller-type diffusion process $\left\{X(t), t \geq t_{0}\right\}, t_{0} \geq 0$, with infinitesimal drift $A_{1}(x, t)=\alpha(t) x+\beta(t)$ and infinitesimal variance $A_{2}(x, t)=2 r(t) x$, defined in the state-space $[0,+\infty)$, with $\alpha(t) \in \mathbb{R}, \beta(t) \in \mathbb{R}$, 

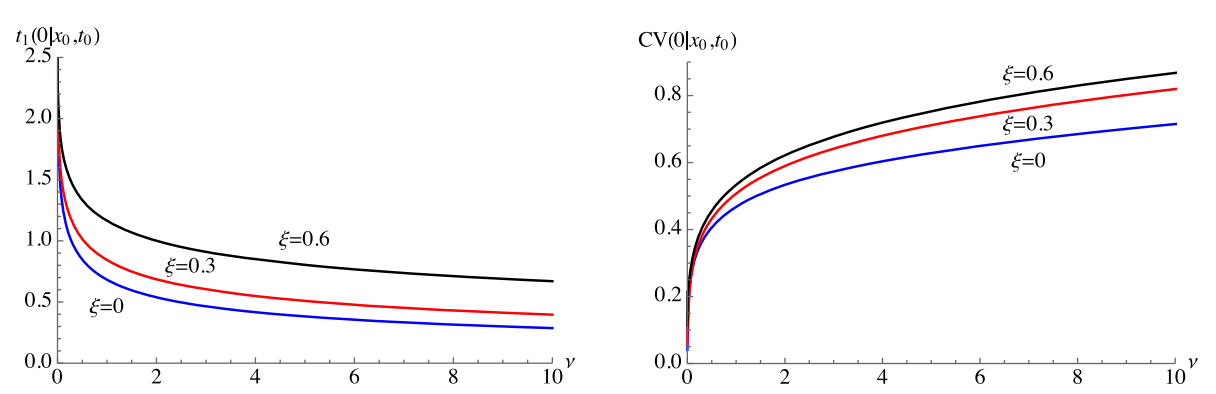

Fig. 9 The mean (on the left) and the coefficient of variation (on the right) of FPT from $X(0)=5$ to the zero-state are plotted as function of $v$ for the process (60), being $r(t)$ defined in (54), with $c=0.9$ and $Q=2$, and $\alpha(t)$ given in (57), with $\eta=-5, b=0.3$ and $Q_{1}=1$

$r(t)>0, \beta(t) \leq \xi r(t)$, with $0 \leq \xi<1$, for all $t \geq t_{0}$. We have assumed that the zero-state represents an absorbing boundary for $X(t)$. This process plays a relevant role in different fields, including physics, biology, neuroscience, finance and others. For instance, in population biology $\alpha(t)$ represents the growth intensity function and can be positive, negative or zero at different time instants, $\beta(t)$ describes the immigration intensity function; instead, the noise intensity function $r(t)$ takes into account the environmental fluctuations. For this process, the transition density $f_{a}\left(x, t \mid x_{0}, t_{0}\right)$ in the presence of an absorbing boundary in zero-state and the FPT density $g\left(0, t \mid x_{0}, t_{0}\right)$ from $X\left(t_{0}\right)=x_{0}$ to the zero-state are obtained. Special attention is dedicated to the proportional case, in which the immigration intensity function and the noise intensity function are related as $\beta(t)=\xi r(t)$, with $0 \leq \xi<1$. Various numerical computation are performed to illustrate the effect of periodic intensity functions on the FPT pdf $g\left(0, t \mid x_{0}, t_{0}\right)$, by assuming that $\alpha(t), \beta(t)$ or both these functions exhibit some kind of periodicity.

Acknowledgements This research is partially supported by MIUR - PRIN 2017, Project "Stochastic Models for Complex Systems". The authors are members of the group GNCS of INdAM.

Funding : Open access funding provided by University degli Studi di Salerno within the CRUI-CARE Agreement.

Open Access This article is licensed under a Creative Commons Attribution 4.0 International License, which permits use, sharing, adaptation, distribution and reproduction in any medium or format, as long as you give appropriate credit to the original author(s) and the source, provide a link to the Creative Commons licence, and indicate if changes were made. The images or other third party material in this article are included in the article's Creative Commons licence, unless indicated otherwise in a credit line to the material. If material is not included in the article's Creative Commons licence and your intended use is not permitted by statutory regulation or exceeds the permitted use, you will need to obtain permission directly from the copyright holder. To view a copy of this licence, visit http://creativecommons.org/licenses/by/4.0/.

\section{Appendix}

\section{A Proof of Proposition 1}

To solve (10) with initial condition (11), we use the method of characteristics (cf., for instance, Williams [46]) and we consider the following differential equations: 


$$
\frac{d t_{0}}{d \xi}=1, \quad \frac{d s}{d \xi}=-s\left[\alpha\left(t_{0}\right)+s r\left(t_{0}\right)\right], \quad \frac{d Z_{a}}{d \xi}=\left[\alpha\left(t_{0}\right)+2 s r\left(t_{0}\right)-s \beta\left(t_{0}\right)\right] Z_{a},
$$

with the initial conditions:

$$
t_{0}(w, \xi=t)=t, \quad s(w, \xi=t)=w, \quad Z_{a}(w, \xi=t)=e^{-w x} .
$$

The first equation of (A1), with the related initial condition in (A2), leads to $t_{0}=\xi$. Then, solving the second equation in (A1) with $t_{0}=\xi$ and making use of the second of (A2), one has:

$$
s=\frac{w e^{-A(\xi \mid t)}}{1+w R(\xi \mid t)} .
$$

Moreover, solving the third equation in (A1) with $t_{0}=\xi$ and $s$ given in (3), we have

$$
Z_{a}(w, \xi)=e^{-w x} \exp \left\{A(\xi \mid t)+\int_{t}^{\xi}[2 r(u)-\beta(u)] \frac{w e^{-A(u \mid t)}}{1+w R(u \mid t)} d u\right\},
$$

where the third of (A2) has been used. From (3) with $\xi=t_{0}$, we also obtain

$$
w=\frac{s e^{-A\left(t \mid t_{0}\right)}}{1+s R\left(t \mid t_{0}\right)} .
$$

Hence, recalling that $\xi=t_{0}$ and making use of (5), from (4) it follows:

$$
\begin{aligned}
& Z_{a}\left(x, t \mid s, t_{0}\right)=e^{-A\left(t \mid t_{0}\right)} \exp \left\{-\frac{s x e^{-A\left(t \mid t_{0}\right)}}{1+s R\left(t \mid t_{0}\right)}\right\} \\
& \quad \times \exp \left\{-\int_{t_{0}}^{t}[2 r(u)-\beta(u)] \frac{s e^{-A\left(u \mid t_{0}\right)}}{1+s\left[R\left(t \mid t_{0}\right)+e^{-A\left(t \mid t_{0}\right)} R(u \mid t)\right]} d u\right\} .
\end{aligned}
$$

Since

$$
R\left(t \mid t_{0}\right)+e^{-A\left(t \mid t_{0}\right)} R(u \mid t)=R\left(u \mid t_{0}\right), \quad t_{0} \leq u \leq t,
$$

one has:

$$
\begin{aligned}
& \exp \left\{-\int_{t_{0}}^{t}[2 r(u)-\beta(u)] \frac{s e^{-A\left(u \mid t_{0}\right)}}{1+s\left[R\left(t \mid t_{0}\right)+e^{-A\left(t \mid t_{0}\right)} R(u \mid t)\right]} d u\right\} \\
& =\exp \left\{-2 \int_{t_{0}}^{t} r(u) \frac{s e^{-A\left(u \mid t_{0}\right)}}{1+s R\left(u \mid t_{0}\right)} d u\right\} \exp \left\{\int_{t_{0}}^{t} \beta(u) \frac{s e^{-A\left(u \mid t_{0}\right)}}{1+s R\left(u \mid t_{0}\right)} d u\right\} .
\end{aligned}
$$

We note that

$$
\exp \left\{-2 \int_{t_{0}}^{t} r(u) \frac{s e^{-A\left(u \mid t_{0}\right)}}{1+s R\left(u \mid t_{0}\right)} d u\right\}=\frac{1}{\left[1+s R\left(t \mid t_{0}\right)\right]^{2}}
$$

being

$$
\frac{d}{d u} \ln \left[1+s R\left(u \mid t_{0}\right)\right]=\frac{s r(u) e^{-A\left(u \mid t_{0}\right)}}{1+s R\left(u \mid t_{0}\right)}, \quad t_{0} \leq u \leq t .
$$

Making use of (7) and (8) in (6), one obtains (12). Finally, we note that the assumptions on the functions $\alpha(t), \beta(t)$ and $r(t)$ in Proposition 1 imply that

$$
0 \leq Z_{a}\left(x, t \mid s, t_{0}\right) \leq \frac{e^{-A\left(t \mid t_{0}\right)}}{\left[1+s R\left(t \mid t_{0}\right)\right]^{2-\xi}} \exp \left\{-\frac{s x e^{-A\left(t \mid t_{0}\right)}}{1+s R\left(t \mid t_{0}\right)}\right\}, \quad 0 \leq \xi<1,
$$


so that

$$
\lim _{x_{0} \downarrow 0} f_{a}\left(x, t \mid x_{0}, t_{0}\right)=\lim _{s \uparrow+\infty} s Z_{a}\left(x, t \mid s, t_{0}\right)=0,
$$

i.e. the condition (5) is satisfied.

\section{B Proof of Proposition 2}

We note that

$$
\exp \left\{\int_{t_{0}}^{t} \beta(u) \frac{s e^{-A\left(u \mid t_{0}\right)}}{1+s R\left(u \mid t_{0}\right)} d u\right\}=\exp \left\{\xi \int_{t_{0}}^{t} \frac{s r(u) e^{-A\left(u \mid t_{0}\right)}}{1+s R\left(u \mid t_{0}\right)} d u\right\}=\left[1+s R\left(t \mid t_{0}\right)\right]^{\xi},
$$

where the last identity follows by virtue of (9). Hence, making use of (B1) in (12), one obtains (15). To derive (16), we consider the inverse Laplace transform of (15) distinguishing two cases: (i) $x=0$ and (ii) $x>0$.

Case (i) If $x=0$, Eq. (15) becomes:

$$
Z_{a}\left(0, t \mid s, t_{0}\right)=\frac{e^{-A\left(t \mid t_{0}\right)}}{\left[R\left(t \mid t_{0}\right)\right]^{2-\xi}}\left[s+\frac{1}{R\left(t \mid t_{0}\right)}\right]^{\xi-2}, \quad 0 \leq \xi<1 .
$$

Since (cf. Erdèlyi et al. [43], p. 144, no. 3)

$$
\int_{0}^{+\infty} e^{-s x_{0}} x_{0}^{\nu-1} e^{-a x_{0}} d x_{0}=\Gamma(v)(s+a)^{-v}, \quad \operatorname{Re} v>0,
$$

taking the inverse Laplace transform in (B2), for $t \geq t_{0}$ the first of (16) immediately follows. Case (ii) Let $x_{0}>0$. By setting

$$
1+s R\left(t \mid t_{0}\right)=z, \quad \frac{x_{0}}{R\left(t \mid t_{0}\right)}=y,
$$

in (15), making use of (9), one has:

$$
\begin{aligned}
& \int_{0}^{+\infty} e^{-z y}\left\{e^{y} f_{a}\left[x, t \mid R\left(t \mid t_{0}\right) y, t_{0}\right]\right\} d y=\frac{e^{-A\left(t \mid t_{0}\right)}}{R\left(t \mid t_{0}\right)} \exp \left\{-\frac{x e^{-A\left(t \mid t_{0}\right)}}{R\left(t \mid t_{0}\right)}\right\} \\
& \times z^{\xi-2} \exp \left\{\frac{x e^{-A\left(t \mid t_{0}\right)}}{z R\left(t \mid t_{0}\right)}\right\}, \quad 0 \leq \xi<1 .
\end{aligned}
$$

Since (cf. Erdèlyi et al. [43], p. 197, no. 18)

$$
\int_{0}^{+\infty} e^{-z y} a^{-v / 2} y^{v / 2} I_{v}\left(2 \sqrt{a y)} d y=z^{-v-1} e^{a / z}, \quad \operatorname{Re} v>-1,\right.
$$

taking the inverse Laplace transform in (B4), for $t \geq t_{0}$ one obtains:

$$
\begin{aligned}
& f_{a}\left[x, t \mid R\left(t \mid t_{0}\right) y, t_{0}\right]=e^{-y} \frac{e^{-A\left(t \mid t_{0}\right)}}{R\left(t \mid t_{0}\right)} \exp \left\{-\frac{x e^{-A\left(t \mid t_{0}\right)}}{R\left(t \mid t_{0}\right)}\right\}\left[\frac{x e^{-A\left(t \mid t_{0}\right)}}{R\left(t \mid t_{0}\right)}\right]^{-(1-\xi) / 2} \\
& \times y^{(1-\xi) / 2} I_{1-\xi}\left[2 \sqrt{\frac{x y e^{-A\left(t \mid t_{0}\right)}}{R\left(t \mid t_{0}\right)}}\right], \quad 0 \leq \xi<1,
\end{aligned}
$$

from which, applying again the transformation $x_{0}=R\left(t \mid t_{0}\right) y$, the second of (16) follows. 


\section{Proof of Proposition 5}

Let $x_{0}>0$ and $t \geq t_{0}$. Making use of (B3) in (30) and recalling (9), one has:

$$
\begin{aligned}
& \int_{0}^{+\infty} e^{-z y}\left\{e^{y} f_{a}\left[0, t \mid R\left(t \mid t_{0}\right) y, t_{0}\right]\right\} d y=\frac{e^{-A\left(t \mid t_{0}\right)}}{R\left(t \mid t_{0}\right) z^{2}} \\
& \quad \times \exp \left\{(z-1) \int_{t_{0}}^{t} \frac{\beta(u) e^{-A\left(u \mid t_{0}\right)}}{R\left(t \mid t_{0}\right)+(z-1) R\left(u \mid t_{0}\right)} d u\right\} .
\end{aligned}
$$

We note that

$$
\begin{aligned}
& \exp \left\{(z-1) \int_{t_{0}}^{t} \frac{\beta(u) e^{-A\left(u \mid t_{0}\right)}}{R\left(t \mid t_{0}\right)+(z-1) R\left(u \mid t_{0}\right)} d u\right\} \\
& =\exp \left\{\frac{z-1}{z} \int_{t_{0}}^{t} \frac{\beta(u) e^{-A\left(u \mid t_{0}\right)}}{R\left(t \mid t_{0}\right)\left[1-\left(\frac{z-1}{z}\right)\left(1-\frac{R\left(u \mid t_{0}\right)}{R\left(t \mid t_{0}\right)}\right)\right]} d u\right\} \\
& =\exp \left\{\sum_{k=1}^{+\infty}\left(1-\frac{1}{z}\right)^{k} \frac{1}{\left[R\left(t \mid t_{0}\right)\right]^{k}} \int_{t_{0}}^{t} \beta(u) e^{-A\left(u \mid t_{0}\right)}\left[R\left(t \mid t_{0}\right)-R\left(u \mid t_{0}\right)\right]^{k-1} d u\right\},
\end{aligned}
$$

where the last equality follows being

$$
0<\frac{z-1}{z}\left(1-\frac{R\left(u \mid t_{0}\right)}{R\left(t \mid t_{0}\right)}\right)<1, \quad t_{0} \leq u \leq t .
$$

Since (cf., for instance, Comtet [47]):

$$
\exp \left\{\sum_{r=1}^{+\infty} \frac{d_{r}}{r !} \vartheta^{r}\right\}=\sum_{n=0}^{+\infty} \frac{B_{n}\left(d_{1}, d_{2}, \ldots, d_{n}\right)}{n !} \vartheta^{n},
$$

where $B_{n}\left(d_{1}, d_{2}, \ldots, d_{n}\right)$ are the complete Bell polynomials defined in (31), with $d_{k}$ given in (32), from (C2) one obtains:

$$
\exp \left\{(z-1) \int_{t_{0}}^{t} \frac{\beta(u) e^{-A\left(u \mid t_{0}\right)}}{R\left(t \mid t_{0}\right)+(z-1) R\left(u \mid t_{0}\right)} d u\right\}=\sum_{n=0}^{+\infty} \frac{B_{n}\left(d_{1}, d_{2}, \ldots, d_{n}\right)}{n !}\left(1-\frac{1}{z}\right)^{n}
$$

Then, making use of (C3) in (C1) one has:

$$
\int_{0}^{+\infty} e^{-z y}\left\{e^{y} f_{a}\left[0, t \mid R\left(t \mid t_{0}\right) y, t_{0}\right]\right\} d y=\frac{e^{-A\left(t \mid t_{0}\right)}}{R\left(t \mid t_{0}\right) z^{2}} \sum_{n=0}^{+\infty} \frac{B_{n}\left(d_{1}, d_{2}, \ldots, d_{n}\right)}{n !}\left(\frac{z-1}{z}\right)^{n} .
$$

Finally, since (cf. Gradshteyn and Ryzhik [48], p. 809, no. 8)

$$
\int_{0}^{+\infty} e^{-z y} y^{\alpha} L_{n}^{(\alpha)}(y) d y=\frac{\Gamma(\alpha+n+1)(z-1)^{n}}{n ! z^{\alpha+n+1}} \quad \operatorname{Re} \alpha>-1, \operatorname{Re} z>0,
$$


by setting $\alpha=1$, Eq. (C4) leads to:

$$
f_{a}\left[0, t \mid R\left(t \mid t_{0}\right) y, t_{0}\right]=e^{-y} \frac{e^{-A\left(t \mid t_{0}\right)}}{R\left(t \mid t_{0}\right)} \sum_{n=0}^{+\infty} \frac{B_{n}\left(d_{1}, d_{2}, \ldots, d_{n}\right)}{n !} \frac{y}{n+1} L_{n}^{(1)}(y), \quad y>0 .
$$

Applying again the transformation $x_{0}=R\left(t \mid t_{0}\right) y$, one obtains (33).

\section{Proof of Proposition 6}

We use (B3) in (29), so that, by virtue of (39), for $t \geq t_{0}$ we obtain:

$$
\begin{aligned}
& \int_{0}^{+\infty} e^{-z y}\left\{e^{y} v_{a}\left[x, t \mid R\left(t \mid t_{0}\right) y, t_{0}\right]\right\} d y=\frac{1}{R\left(t \mid t_{0}\right)} \exp \left\{-\frac{x e^{-A\left(t \mid t_{0}\right)}}{R\left(t \mid t_{0}\right)}\right\} \exp \left\{\frac{x e^{-A\left(t \mid t_{0}\right)}}{R\left(t \mid t_{0}\right) z}\right\} \\
& \quad y>0, x>0 .
\end{aligned}
$$

Since (cf. Erdèlyi et al. [43], p. 197, no. 16)

$$
\int_{0}^{+\infty} e^{-z y}\left[\delta(y)+\frac{\sqrt{a} I_{1}(2 \sqrt{a y})}{\sqrt{y}}\right] d y=e^{a / z}, \quad \operatorname{Re} z>0, \operatorname{Re} a>0,
$$

from (D1) for $t \geq t_{0}$ and $x>0$ one has:

$$
\begin{aligned}
& v_{a}\left[x, t \mid R\left(t \mid t_{0}\right) y, t_{0}\right]=\frac{e^{-y}}{R\left(t \mid t_{0}\right)} \exp \left\{-\frac{x e^{-A\left(t \mid t_{0}\right)}}{R\left(t \mid t_{0}\right)}\right\} \\
& \quad \times\left\{\delta(y)+\sqrt{\frac{x e^{-A\left(t \mid t_{0}\right)}}{y R\left(t \mid t_{0}\right)}} I_{1}\left[2 \sqrt{\frac{x y e^{-A\left(t \mid t_{0}\right)}}{R\left(t \mid t_{0}\right)}}\right]\right\}, \quad y>0 .
\end{aligned}
$$

Then, applying the transformation $x_{0}=R\left(t \mid t_{0}\right) y$, Eq. (40) follows from (D2), recalling that $\delta(a x)=\delta(x) /|a|$ and $g(x) \delta(x-a)=g(a) \delta(x-a)$.

\section{E Proof of Proposition 8}

From (42), we obtain:

$$
\begin{aligned}
& \int_{0}^{+\infty} f_{a}\left(x, t \mid x_{0}, t_{0}\right) d x=\left[\frac{1}{R\left(t \mid t_{0}\right)}\right]^{3 / 2} \exp \left\{-\frac{x_{0}}{R\left(t \mid t_{0}\right)}\right\}\left\{x_{0} \sqrt{R\left(t \mid t_{0}\right)} \Psi\left(t \mid x_{0}, t_{0}\right)\right. \\
& \left.+\int_{0}^{x_{0}} d z \frac{z \Psi\left(t \mid z, t_{0}\right)}{\sqrt{x_{0}-z}} \int_{0}^{+\infty} e^{-y} \sqrt{y} I_{1}\left[2 \frac{\sqrt{x_{0}-z}}{R\left(t \mid t_{0}\right)} \sqrt{y}\right] d y\right\} .
\end{aligned}
$$

We note that (cf. Erdèlyi et al [43], p. 197, no. 18)

$$
\int_{0}^{+\infty} e^{-p y} y^{v / 2} I_{\nu}(2 \sqrt{a y}) d y=a^{v / 2} p^{-v-1} e^{a / p}, \quad \operatorname{Re} p>0, \operatorname{Re} v>-1,
$$

so that from (E1), by virtue of (34), it follows:

$$
\begin{aligned}
& \int_{0}^{+\infty} f_{a}\left(x, t \mid x_{0}, t_{0}\right) d x=\frac{1}{R\left(t \mid t_{0}\right)}\left[x_{0} \exp \left\{-\frac{x_{0}}{R\left(t \mid t_{0}\right)}\right\} \Psi\left(t \mid x_{0}, t_{0}\right)\right. \\
& \left.+R\left(t \mid t_{0}\right) \sum_{n=0}^{+\infty} \frac{B_{n}\left(d_{1}, d_{2}, \ldots, d_{n}\right)}{(n+1) !} \int_{0}^{x_{0} / R\left(t \mid t_{0}\right)} y e^{-y} L_{n}^{(1)}(y) d y\right] .
\end{aligned}
$$


Recalling the expression of the Laguerre polynomials (35), one has:

$$
\int_{0}^{z} y e^{-y} L_{n}^{(1)}(y) d y= \begin{cases}1-(1+z) e^{-z}, & n=0, \\ z^{2} e^{-z}, & n=1, \\ \frac{z^{2} e^{-z}}{n} L_{n-1}^{(2)}(z), & n=2,3, \ldots\end{cases}
$$

Then, making use of (E3) in (E2), for $t \geq t_{0}$ and $x_{0}>0$ one obtains:

$$
\begin{gathered}
\int_{0}^{+\infty} f_{a}\left(x, t \mid x_{0}, t_{0}\right) d x=1-\exp \left\{-\frac{x_{0}}{R\left(t \mid t_{0}\right)}\right\}+\frac{x_{0}}{R\left(t \mid t_{0}\right)} \exp \left\{-\frac{x_{0}}{R\left(t \mid t_{0}\right)}\right\} \\
\times \sum_{n=1}^{+\infty} \frac{B_{n}\left(d_{1}, d_{2}, \ldots, d_{n}\right)}{(n+1) !}\left\{L_{n}^{(1)}\left[\frac{x_{0}}{R\left(t \mid t_{0}\right)}\right]+\frac{1}{n} \frac{x_{0}}{R\left(t \mid t_{0}\right)} L_{n-1}^{(2)}\left[\frac{x_{0}}{R\left(t \mid t_{0}\right)}\right]\right\} .
\end{gathered}
$$

Moreover, since the Laguerre polynomials satisfy the following functional relations (cf. Gradshteyn and Ryzhik [48], p. 1001, no. 8.971.4 and no. 8.971.5)

$$
\begin{aligned}
& y L_{n}^{(a+1)}(z)=(n+a) L_{n-1}^{(a)}(z)-(n-y) L_{n}^{(a)}(z), \\
& L_{n}^{(a-1)}(z)=L_{n}^{(a)}(z)-L_{n-1}^{(a)}(z),
\end{aligned}
$$

one also has:

$$
n L_{n}^{(1)}(z)+y L_{n-1}^{(2)}(z)=(n+1) L_{n-1}^{(1)}(z) .
$$

Hence, (E4) can be rewritten as

$$
\begin{aligned}
& \int_{0}^{+\infty} f_{a}\left(x, t \mid x_{0}, t_{0}\right) d x=1-\exp \left\{-\frac{x_{0}}{R\left(t \mid t_{0}\right)}\right\}+\frac{x_{0}}{R\left(t \mid t_{0}\right)} \exp \left\{-\frac{x_{0}}{R\left(t \mid t_{0}\right)}\right\} \\
& \times \sum_{n=1}^{+\infty} \frac{B_{n}\left(d_{1}, d_{2}, \ldots, d_{n}\right)}{n n !} L_{n-1}^{(1)}\left[\frac{x_{0}}{R\left(t \mid t_{0}\right)}\right] .
\end{aligned}
$$

Finally, since (cf. Gradshteyn and Ryzhik [48], p. 1001, no. 8.972.1)

$$
L_{n}^{(a)}(z)=\left(\begin{array}{c}
n+a \\
n
\end{array}\right) \Phi(-n, a+1 ; z), \quad a \geq 0, n=0,1, \ldots
$$

Eq. (44) follows immediately from (E5). 


\section{F Proof of Proposition 9}

Making use of (44) in (50), one has

$$
\begin{aligned}
& g\left(0, t \mid x_{0}, t_{0}\right)=\frac{x_{0}}{R\left(t \mid t_{0}\right)} \exp \left\{-\frac{x_{0}}{R\left(t \mid t_{0}\right)}\right\}\left\{\frac{r(t) e^{-A\left(t \mid t_{0}\right)}}{R\left(t \mid t_{0}\right)}\right. \\
& \times\left[1+\left(1-\frac{x_{0}}{R\left(t \mid t_{0}\right)}\right) \sum_{n=1}^{+\infty} \frac{B_{n}\left(d_{1}, d_{2}, \ldots, d_{n}\right)}{n !} \Phi\left(1-n, 2 ; \frac{x_{0}}{R\left(t \mid t_{0}\right)}\right)\right] \\
& -\sum_{n=1}^{+\infty} \frac{B_{n}\left(d_{1}, d_{2}, \ldots, d_{n}\right)}{n !} \frac{d}{d t} \Phi\left(1-n, 2 ; \frac{x_{0}}{R\left(t \mid t_{0}\right)}\right) \\
& -\sum_{n=1}^{+\infty} \frac{1}{n !} \Phi\left(1-n, 2 ; \frac{x_{0}}{R\left(t \mid t_{0}\right)}\right) \frac{d}{d t} B_{n}\left(d_{1}, d_{2}, \ldots, d_{n}\right) .
\end{aligned}
$$

Since (cf. Gradshteyn and Ryzhik [48], p. 1023, no. 9213)

$$
\frac{d}{d z} \Phi(a, b ; z)=\frac{a}{b} \Phi(a+1, b+1 ; z),
$$

one obtains:

$$
\frac{d}{d t} \Phi\left(1-n, 2 ; \frac{x_{0}}{R\left(t \mid t_{0}\right)}\right)=\frac{n-1}{2} \frac{x_{0} r(t) e^{-A\left(t \mid t_{0}\right)}}{R^{2}\left(t \mid t_{0}\right)} \Phi\left(2-n, 3 ; \frac{x_{0}}{R\left(t \mid t_{0}\right)}\right) .
$$

Therefore, Eq. (F1) can be rewritten as:

$$
\begin{aligned}
& g\left(0, t \mid x_{0}, t_{0}\right)=\frac{x_{0}}{R\left(t \mid t_{0}\right)} \exp \left\{-\frac{x_{0}}{R\left(t \mid t_{0}\right)}\right\}\left\{\frac{r(t) e^{-A\left(t \mid t_{0}\right)}}{R\left(t \mid t_{0}\right)} \sum_{n=0}^{+\infty} \frac{B_{n}\left(d_{1}, d_{2}, \ldots, d_{n}\right)}{n !}\right. \\
& \times\left[\left(1-\frac{x_{0}}{R\left(t \mid t_{0}\right)}\right) \Phi\left(1-n, 2 ; \frac{x_{0}}{R\left(t \mid t_{0}\right)}\right)-\frac{n-1}{2} \frac{x_{0}}{R\left(t \mid t_{0}\right)} \Phi\left(2-n, 3 ; \frac{x_{0}}{R\left(t \mid t_{0}\right)}\right)\right] \\
& \left.-\sum_{n=1}^{+\infty} \frac{1}{n !} \Phi\left(1-n, 2 ; \frac{x_{0}}{R\left(t \mid t_{0}\right)}\right) \frac{d}{d t} B_{n}\left(d_{1}, d_{2}, \ldots, d_{n}\right)\right\}
\end{aligned}
$$

where the use of the following relations

$$
\begin{aligned}
& \Phi(a, a ; z)=e^{z}, \\
& \frac{z}{b} \Phi(a+1, b+1 ; z)=\Phi(a+1, b ; z)-\Phi(a, b ; z)
\end{aligned}
$$

has been made. Finally, recalling that

$$
a \Phi(a+1, b+1 ; z)=(a-b) \Phi(a, b+1 ; z)+b \Phi(a, b ; z),
$$

the expression in square bracket in Eq. (F2) becomes:

$$
\begin{aligned}
& \left(1-\frac{x_{0}}{R\left(t \mid t_{0}\right)}\right) \Phi\left(1-n, 2 ; \frac{x_{0}}{R\left(t \mid t_{0}\right)}\right)-\frac{n-1}{2} \frac{x_{0}}{R\left(t \mid t_{0}\right)} \Phi\left(2-n, 3 ; \frac{x_{0}}{R\left(t \mid t_{0}\right)}\right) \\
& \quad=\Phi\left(1-n, 1 ; \frac{x_{0}}{R\left(t \mid t_{0}\right)}\right)-\frac{x_{0}}{R\left(t \mid t_{0}\right)} \Phi\left(1-n, 2 ; \frac{x_{0}}{R\left(t \mid t_{0}\right)}\right) \\
& =\Phi\left(-n, 1 ; \frac{x_{0}}{R\left(t \mid t_{0}\right)}\right),
\end{aligned}
$$


where the last identity follows from (F3). Then, substituting (F4) in (F2), we obtain Eq. (51).

\section{G Evaluation of $R\left(t \mid t_{0}\right)$ in (61)}

From (61) one has:

$$
R\left(t \mid t_{0}\right)=\frac{v}{1+b \sin \left(\frac{2 \pi t_{0}}{Q_{1}}\right)}\left[R_{1}\left(t \mid t_{0}\right)+c R_{2}\left(t \mid t_{0}\right)+b c R_{3}\left(t \mid t_{0}\right)\right]
$$

with $0 \leq b<1$ and $0 \leq c<1$, where

$$
\begin{aligned}
& R_{1}\left(t \mid t_{0}\right)=\int_{t_{0}}^{t} e^{-\eta\left(\tau-t_{0}\right)}\left[1+b \sin \left(\frac{2 \pi \tau}{Q_{1}}\right)\right] d \tau \\
& =\left\{\begin{array}{l}
t-t_{0}-\frac{b Q_{1}}{2 \pi}\left[\cos \left(\frac{2 \pi t}{Q_{1}}\right)-\cos \left(\frac{2 \pi t_{0}}{Q_{1}}\right)\right], \\
\frac{1-e^{-\eta\left(t-t_{0}\right)}}{\eta}-\frac{2 \pi b Q_{1}}{4 \pi^{2}+Q_{1}^{2} \eta^{2}}\left[e^{-\eta\left(t-t_{0}\right)} \cos \left(\frac{2 \pi t}{Q_{1}}\right)\right. \\
\left.+\frac{Q_{1} \eta}{2 \pi} e^{-\eta\left(t-t_{0}\right)} \sin \left(\frac{2 \pi t}{Q_{1}}\right)-\cos \left(\frac{2 \pi t_{0}}{Q_{1}}\right)-\frac{Q_{1} \eta}{2 \pi} \sin \left(\frac{2 \pi t_{0}}{Q_{1}}\right)\right], \eta \neq 0,
\end{array}\right. \\
& R_{2}\left(t \mid t_{0}\right)=\int_{t_{0}}^{t} e^{-\eta\left(\tau-t_{0}\right)} \sin \left(\frac{2 \pi \tau}{Q}\right) d \tau \\
& =\left\{\begin{array}{l}
\frac{Q}{2 \pi}\left[\cos \left(\frac{2 \pi t_{0}}{Q}\right)-\cos \left(\frac{2 \pi t}{Q}\right)\right], \\
\frac{2 \pi Q}{4 \pi^{2}+Q^{2} \eta^{2}}\left\{\cos \left(\frac{2 \pi t_{0}}{Q}\right)+\frac{Q \eta}{2 \pi} \sin \left(\frac{2 \pi t_{0}}{Q}\right)\right. \\
\left.-e^{-\eta\left(t-t_{0}\right)}\left[\cos \left(\frac{2 \pi t}{Q}\right)+\frac{Q \eta}{2 \pi} \sin \left(\frac{2 \pi t}{Q}\right)\right]\right\}, \eta \neq 0,
\end{array}\right.
\end{aligned}
$$

and

$$
\begin{aligned}
& R_{3}\left(t \mid t_{0}\right)=\int_{t_{0}}^{t} e^{-\eta\left(\tau-t_{0}\right)} \sin \left(\frac{2 \pi \tau}{Q}\right) \sin \left(\frac{2 \pi \tau}{Q_{1}}\right) d \tau \\
& \begin{array}{ll}
\frac{Q Q_{1}}{4 \pi}\left\{\frac{1}{Q-Q_{1}}\left[\sin \left(2 \pi t \frac{Q-Q_{1}}{Q Q_{1}}\right)-\sin \left(2 \pi t_{0} \frac{Q-Q_{1}}{Q Q_{1}}\right)\right]\right. \\
\left.-\frac{1}{Q+Q_{1}}\left[\sin \left(2 \pi t \frac{Q+Q_{1}}{Q Q_{1}}\right)-\sin \left(2 \pi t_{0} \frac{Q+Q_{1}}{Q Q_{1}}\right)\right]\right\}, \\
\frac{Q Q_{1}}{2}\left\{e^{-\eta\left(t-t_{0}\right)\left[\frac{Q Q_{1} \eta \cos \left(2 \pi t \frac{Q+Q_{1}}{Q Q_{1}}\right)-2 \pi\left(Q+Q_{1}\right) \sin \left(2 \pi t \frac{Q+Q_{1}}{Q Q_{1}}\right)}{4 \pi^{2}\left(Q+Q_{1}\right)^{2}+Q^{2} Q_{1}^{2} \eta^{2}}\right.}\right. \\
\left.-\frac{Q Q_{1} \eta \cos \left(2 \pi t \frac{Q-Q_{1}}{Q Q_{1}}\right)-2 \pi\left(Q-Q_{1}\right) \sin \left(2 \pi t \frac{Q-Q_{1}}{Q Q_{1}}\right)}{4 \pi^{2}\left(Q-Q_{1}\right)^{2}+Q^{2} Q_{1}^{2} \eta^{2}}\right] \\
-\frac{Q Q_{1} \eta \cos \left(2 \pi t_{0} \frac{Q+Q_{1}}{Q Q_{1}}\right)-2 \pi\left(Q+Q_{1}\right) \sin \left(2 \pi t_{0} \frac{Q+Q_{1}}{Q Q_{1}}\right)}{4 \pi^{2}\left(Q+Q_{1}\right)^{2}+Q^{2} Q_{1}^{2} \eta^{2}} & \\
\left.+\frac{Q Q_{1} \eta \cos \left(2 \pi t_{0} \frac{Q-Q_{1}}{Q Q_{1}}\right)-2 \pi\left(Q-Q_{1}\right) \sin \left(2 \pi t_{0} \frac{Q-Q_{1}}{Q Q_{1}}\right)}{4 \pi^{2}\left(Q-Q_{1}\right)^{2}+Q^{2} Q_{1}^{2} \eta^{2}}\right\}, & \eta \neq 0,
\end{array}
\end{aligned}
$$


for $Q \neq Q_{1}$, whereas

$$
\begin{aligned}
& R_{3}\left(t \mid t_{0}\right)=\int_{t_{0}}^{t} e^{-\eta\left(\tau-t_{0}\right)} \sin ^{2}\left(\frac{2 \pi \tau}{Q}\right) d \tau \\
& =\left\{\begin{array}{lr}
\frac{t-t_{0}}{2}-\frac{Q}{8 \pi} \sin \left(\frac{4 \pi t}{Q}\right)+\frac{Q}{8 \pi} \sin \left(\frac{4 \pi t_{0}}{Q}\right), & \eta=0, \\
\frac{1-e^{-\eta\left(t-t_{0}\right)}}{2 \eta}+\frac{Q}{2\left[16 \pi^{2}+Q^{2} \eta^{2}\right]}\left\{4 \pi \sin \left(\frac{4 \pi t_{0}}{Q}\right)-Q \pi \cos \left(\frac{4 \pi t_{0}}{Q}\right)\right. & \\
\left.-e^{-\eta\left(t-t_{0}\right)}\left[4 \pi \sin \left(\frac{4 \pi t}{Q}\right)-Q \pi \cos \left(\frac{4 \pi t}{Q}\right)\right]\right\}, & \eta \neq 0,
\end{array}\right.
\end{aligned}
$$

for $Q=Q_{1}$.

\section{References}

1. Giorno, V., Nobile, A.G.: On the construction on a special class of time-inhomogeneous diffusion processes. J. Stat. Phys. 177(2), 299-323 (2019)

2. Giorno, V., Nobile, A.G.: Restricted Gompertz-type diffusion processes with periodic regulation functions. Mathematics 7, 555 (2019)

3. Albano, G., Giorno, V.: Inference on the effect of non homogeneous inputs in Ornstein-Uhlenbeck neuronal modeling. Math. Biosci. Eng. 17(1), 328-348 (2019)

4. Ghost, H.: Prajneshu: Gompertz growth model in random environment with time-dependent diffusion. J. Stat. Theory Pract. 11, 746-758 (2017)

5. Buonocore, A., Caputo, L., Nobile, A.G., Pirozzi, E.: Restricted Ornstein-Uhlenbeck process and applications in neuronal models with periodic input signals. J. Comp. Appl. Math. 285, 59-71 (2015)

6. Gutiérrez, R., Ricciardi, L.M., Román, P., Torres, F.: First-passage-time densities for time-nonhomogeneous diffusion processes. J. Appl. Prob. 34(3), 623-631 (1997)

7. Di Crescenzo, A., Giorno, V., Krishna Kumar, B., Nobile, A.G.: A time-non-homogeneous double-ended queue with failures and repairs and its continuous approximation. Mathematics 6(5), 81 (2018)

8. Román-Román, P., Serrano-Pérez, J.J., Torres-Ruiz, F.: Fitting real data by means of non-homogeneous lognormal diffusion processes. Stat. Interface 10, 587-600 (2017)

9. Molini, A., Talkner, P., Katul, G.G., Porporato, A.: First passage time statistics of Brownian motion with purely time dependent drift and diffusion. Physica A 390, 1841-1852 (2011)

10. Gan, X., Waxman, D.: Singular solution of the Feller diffusion equation via a spectral decomposition. Phys. Rev. E Stat. Nonlinear Soft. Matter Phys. 19(1), 012123 (2015)

11. Abundo, M.: On the first-passage times of certain Gaussian processes, and related asymptotics. Stoch. Anal. Appl. (2020) https://doi.org/10.1080/07362994.2020.1843495

12. Feller, W.: Two singular diffusion problems. Ann. Math. 54(1), 173-182 (1951)

13. Feller, W.: The parabolic differential equations and the associated semi-groups of transformations. Ann. Math. 55, 468-518 (1952)

14. Peskir, G.: On boundary behaviour of one-dimensional diffusions: from Brown to Feller and beyond. In: Schilling, R.L., Vondraček, Z., Woyczynski, W.A. (eds.) William Feller, Selected Papers II, Springer, 77-93 (2015)

15. Karlin, S., Taylor, H.W.: A Second Course in Stochastic Processes. Academic Press, New York (1981)

16. Giorno, V., Nobile, A.G., Ricciardi, L.M., Sacerdote, L.: Some remarks on the Rayleigh process. J. Appl. Prob. 23(2), 398-408 (1986)

17. Capocelli, R.M., Ricciardi, L.M.: On the transformation of diffusion processes into the Feller process. Math. Biosci. 29, 219-234 (1976)

18. Lavigne, F., Roques, L.: Extinction times of an inhomogeneous Feller diffusion process: a PDF approach. Expo. Math. (2020). https://doi.org/10.1016/j.exmath.2019.12.002

19. Masoliver, J.: Nonstationary Feller process with time-varying coefficients. Phys. Rev. E 93(012122), 1-11 (2016)

20. Ricciardi, L.M., Di Crescenzo, A., Giorno, V., Nobile, A.G.: An outline of theoretical and algorithmic approaches to first passage time problems with applications to biological modeling. Math. Jpn. 50(2), 247-322 (1999)

21. Di Crescenzo, A., Nobile, A.G.: Diffusion approximation to a queueing system with time-dependent arrival and service rates. Queueing Syst. 19, 41-62 (1995) 
22. Ditlevsen, S., Lánský, P.: Estimation of the input parameters in the Feller neuronal model. Phys. Rev. E 73(061910), 1-9 (2006)

23. Lánský, P., Sacerdote, L., Tomassetti, F.: On the comparison of Feller and Ornstein-Uhlenbeck models for neural activity. Biol. Cybern. 73, 457-465 (1995)

24. Nobile, A.G., Pirozzi, E.: On time non-homogeneous Feller-type diffusion process in neuronal modeling. In: Moreno-Díaz, R. et al. (eds.) EUROCAST 2015, LNCS 9520, 183-191 (2015)

25. Giorno, V., Lánský, P., Nobile, A.G., Ricciardi, L.M.: Diffusion approximation and first-passage-time problem for a model neuron. III. A birth-and-death process approach. Biol. Cybern. 58(6), 387-404 (1988)

26. Giorno, V., Nobile, A.G., Ricciardi, L.M.: On neuronal firing modeling via specially confined diffusion processes. Sci. Math. Jpn. 58(2), 265-294 (2003)

27. Buonocore, A., Giorno, V., Nobile, A.G., Ricciardi, L.M.: A neuronal modeling paradigm in the presence of refractoriness. BioSystems 67, 35-43 (2002)

28. Tian, Y., Zhang, H.: Skew CIR process, conditional characteristic function, moments and bond pricing. Appl. Math. Comput. 329, 230-238 (2018)

29. Cox, J.C., Ingersoll, J.E., Jr., Ross, S.A.: A theory of the term structure of interest rates. Econometrica 53, 385-407 (1985)

30. Linetsky, V.: Computing hitting time densities for CIR and OU diffusions. Applications to mean-reverting models. J. Comput. Financ. 7, 1-22 (2004)

31. Göing-Jaeshke, A., Yor, M.: A survey and some generalizations of Bessel processes. Bernoulli 9(2), 313-349 (2003)

32. Giorno, V., Nobile, A.G.: Bell polynomial approach for time-inhomogeneous linear birth-death process with immigration. Mathematics 8, 1123 (2020)

33. Kolmogoroff, A.: Über die analytischen Methoden in der Wahrscheinlichkeitsrechnung. Math. Ann. 104, 415-458 (1931). https://doi.org/10.1007/BF01457949

34. Dynkin, E.B.: Kolmogorov and the theory of Markov processes. Ann. Prob. 17(3), 822-832 (1989)

35. Masoliver, J., Perelló, J.: First-passage and escape problems in the Feller process. Phys. Rev. E 86, 041116 (2012)

36. Buonocore, A., Caputo, L., Nobile, A.G., Pirozzi, E.: On some time-non-homogeneous linear diffusion processes and related bridges. Sci. Math. Jpn. 76(1), 55-77 (2013)

37. D’Onofrio, G., Lánský, P., Pirozzi, E.: On two diffusion neuronal models with multiplicative noise: the mean first-passage time properties. Chaos 28, 043103 (2018)

38. Giorno, V., Nobile, A.G., Pirozzi, E., Ricciardi, L.M.: On the construction of first-passage-time densities for diffusion processes. Sci. Math. Jpn. 64(2), 277-298 (2006)

39. Giorno, V., Nobile, A.G., Pirozzi, E., Ricciardi, L.M.: FPT densities constructions from OrnsteinUhlenbeck process. In: Trappl, R. (ed.) Cybernetics and Systems, pp. 244-249. Austrian Society for Cybernetic Studies, Vienna, Austria (2008)

40. Albano, G., Giorno, V.: On short-term loan interest rate models: a first passage time approach. Mathematics 6, 70 (2018)

41. Di Nardo, E., D’Onofrio, G.: A cumulant approach for the first-passage-time problem of the Feller squareroot process. Appl. Math. Comput. 391, 125707 (2021)

42. Abramowitz, I.A., Stegun, M.: Handbook of Mathematical Functions. Dover Publications, New York (1972)

43. Erdèlyi, A., Magnus, W., Oberthettinger, F., Tricomi, F.G.: Tables of Integral Transforms, vol. 1. Mc Graw-Hill, New York (1954)

44. Erdèlyi, A., Magnus, W., Oberthettinger, F., Tricomi, F.G.: Higher Trascendental Functions, vol. II. Mc Graw-Hill, New York (1953)

45. Tricomi, F.G.: Funzioni ipergeometriche confluenti. Monografie Matematiche a cura del Consiglio Nazionale delle Ricerche. Edizioni Cremonese, Roma (1954)

46. Williams, W.E.: Partial Differential Equations. Clarendon Press, Oxford (1980)

47. Comtet, L.: Advanced Combinatorics: The Art of Finite and Infinite Expansions. D. Reidel Publishing Company, Dordrecht (1974)

48. Gradshteyn, I.S., Ryzhik, I.M.: Table of Integrals. Series and Products, Academic Press Inc, New York (2014)

Publisher's Note Springer Nature remains neutral with regard to jurisdictional claims in published maps and institutional affiliations. 